\title{
An Extensive Literature Review and New Proposal on Optimal Capacitor Placement in Distribution Systems
}

\author{
S. M. Golam Mostafa ${ }^{*}$, Jai Govind Singh ${ }^{2}$ and H.M. Enamul Haque ${ }^{2}$ \\ ${ }^{1}$ Department of Electrical and Electronics Engineering, International Islamic University, Chittagong, Bangladesh \\ ${ }^{2}$ Department of Energy, Environment and Climate Change, Asian Institute of Technology, SERD, Khlong Luang, Thailand
}

Received: November 30, 2020, Revised: December 17, 2020, Accepted: December 18, 2020, Available Online: December 21, 2020

\begin{abstract}
The main goal of power utilities is to supply reliable and quality power to the end-users and fulfill their total demands at all possible locations. Most of the loads are connected in the distribution systems are inductive. The excessive reactive power demand over the distribution network causes tremendous reactive power losses and changes the voltage profile, hence the system's reliability. Shunt Capacitor Bank (SCB) is widely used in the distribution system for reactive power support, voltage profile, and system performance improvement. But there are some challenges to employ SCB in the distribution network; among them, ensuring the most optimum location and size is a big challenge to get the maximum benefits. Some existing techniques showed better loss reduction but needed either larger SCBs sizes or cause improper node voltage. In this research study, the first section provides an extensive literature review of optimal SCBs placement and sizing. Later on, a new technique called Combinatorial Method has been developed for sizing and sitting of optimal Shunt Capacitors to reduce the distribution loss significantly. The developed method was tested for different case studies using Indian practical 22-bus and IEEE-69-bus network. The results were compared with DSA, Fuzzy GA, and TLBO method and found better distribution feeder loss minimization and voltage profile improvement.
\end{abstract}

Keywords: Distribution feeders; Shunt Capacitor Bank; Distribution Losses; SCBs Sizing and Sitting; Voltage Profile Improvement (VPI); Combinatorial Method.

This work is licensed under a $\underline{\text { Creative Commons Attribution-Non Commercial 4.0 International }}$

\section{Table of Contents}

1. Introduction 151

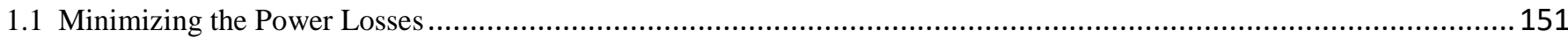

1.2 The Impact of Reactive Power (QCap) in the Vertically Unbundled Electricity Market. ......................................151

1.3 Incorporation of DGs in Distribution System and Reactive Power Management by SCBs .....................................152

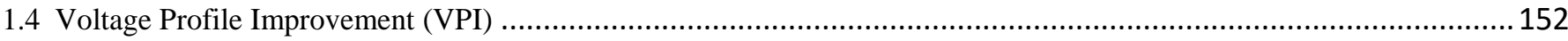

2. Optimum Shunt Capacitor Placement Techniques - A Review ...................................................................... 152

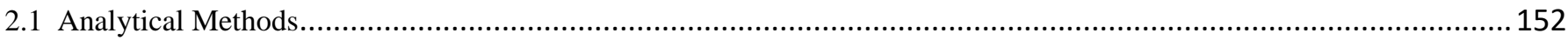

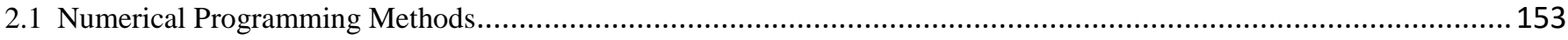

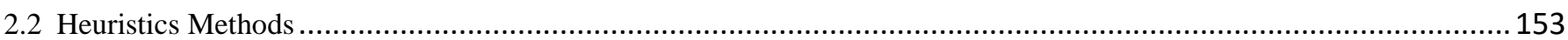

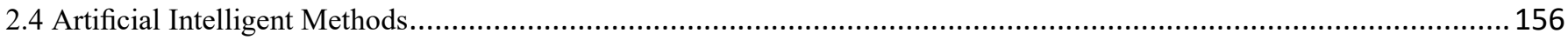

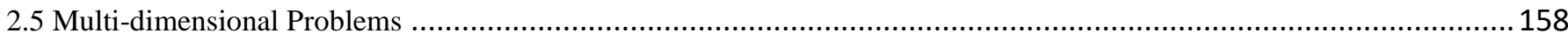

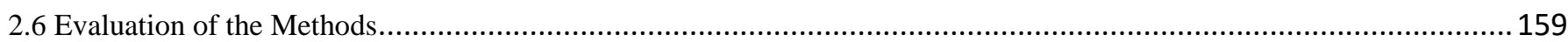

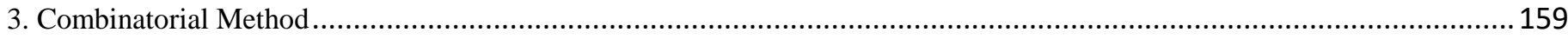

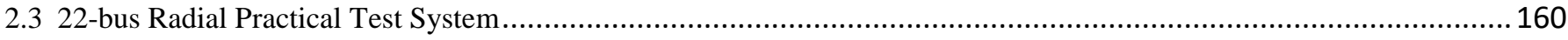

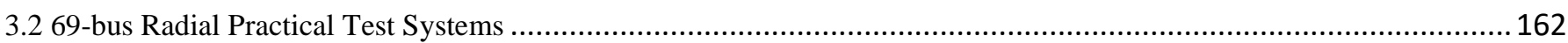

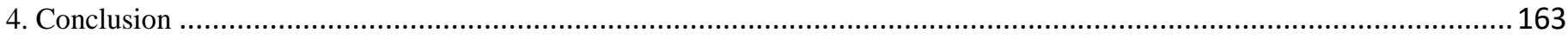

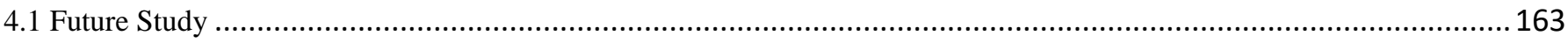

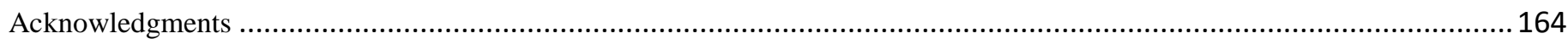

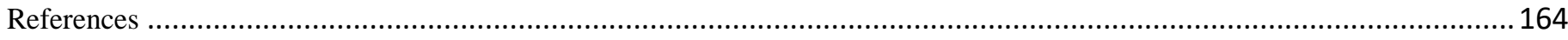




\section{Introduction}

The concept of the electricity market, hence the restructuring and deregulation in existing utilities, had disintegrated the vertically integrated electrical power divisions. The power sectors had unbundled into three main parts: distribution, transmission, and generation sectors. Most power consumers are directly connected to the distribution side though some big customers feed by transmission lines. The reliability and security of distribution feeders are reduced because inductive loads cause greater feeder losses by lagging current. Consequently, any malfunctions or disconnection in any portion of the distribution side will cause a severe effect on reliable and secure power supply in consumer ends. So, it is a vital task of power utilities to reduce the feeder losses and maintain reliability and, hence, the power systems' security. Various FACTS devices and compensators are employed in distribution systems for these reasons [1].

To get the profits of feeder loss minimization, voltage profile enhancements, power factor (p.f.) improvements to a great extent at different scenarios, it is an inevitable task to power engineers to find the optimum placement of Shunt Capacitor Banks (SCBs) with suitable size. To reduce the distribution feeder losses, the SCBs are widely used near the Sub Station (SS). This capacitive compensation reduces the losses and improves the bus voltage and power factor up to the point of common coupling. To achieve a better benefit, it is wise to employ reactive compensating devices at the load center or near the loads. Nowadays, it is possible to connect SCBs at the primary distribution side through available pole-mounted devices and equipment [2]-[6].

In SCBs, the capacitors units are the main building blocks connected in series-parallel combinations in such a manner that keeps over and under voltage limits within $10 \%$ above or below from the nominal values [3]. The total reactive power ( $\left.\mathrm{Q}_{\mathrm{Cap}}\right)$ supplied by the SCBs depends on the capacitive reactance $\left(\mathrm{X}_{\mathrm{Cap}}\right)$ and the supplied voltage $\left(\mathrm{V}_{\mathrm{s}}\right)$ that has been depicted by equation (1) [7]. The recent blackout reported in [8], [9] due to redundancy inadequate reactive power $\left(\mathrm{Q}_{\mathrm{Cap}}\right)$ also draws more attention to manage reactive power $\left(\mathrm{Q}_{\mathrm{Cap}}\right)$ in the system by employing SCBs locally. The researchers proposed a Shunt Capacitor Bank Series Group Shorting (CAPS) method in various low voltage conditions such as generator scheduling, direct load tripping, or in case of line restoration. In this method, the reactive power supplied by shorting various series groups of SCBs units, and these are approximately $20 \%-30 \%$ of the total capacitance of CAPS. The feasibility of CAPS incorporation on High Voltage (HV) and Extra High Voltage (EHV) has been studied in [10]. The optimal allocation of SCBs is the solution of feeder loss minimization, and voltage drop problems can be solved using voltage regulators' placement optimally [4].

$Q_{C a p}=V_{s}^{2} / X_{C a p}$

The necessity of reactive power $\left(\mathrm{Q}_{\mathrm{Cap}}\right)$ in distribution systems can be segregated for the following reasons.

\subsection{Minimizing the Power Losses}

There are two main problems usually found in distribution systems - voltage profile deterioration and higher power losses. Losses in distribution systems are classified as technical and nontechnical losses [11], [12]. Technical losses are losses between the main sub-station to end users through various substation transformers, distribution transformers, primary and secondary lines, voltage regulators, surge arresters. The details of loss measurement have been described in the literature. According to the research conclusion of Energy Information Administration (EIA) and Electric Power Research Institute (EPRI) of America, the distribution losses vary between $33.7 \%$ - 64.9\%. EPRI research shows the distribution losses in Fig. 1. Around $38 \%$ of total distribution losses occurred in primary and 54\% distribution transformers, considering both copper and iron losses, whereas service and secondary loss found 9\%. Fig. 1 depicts that many distribution transformers are the prime reason for higher distribution losses [13], [14].

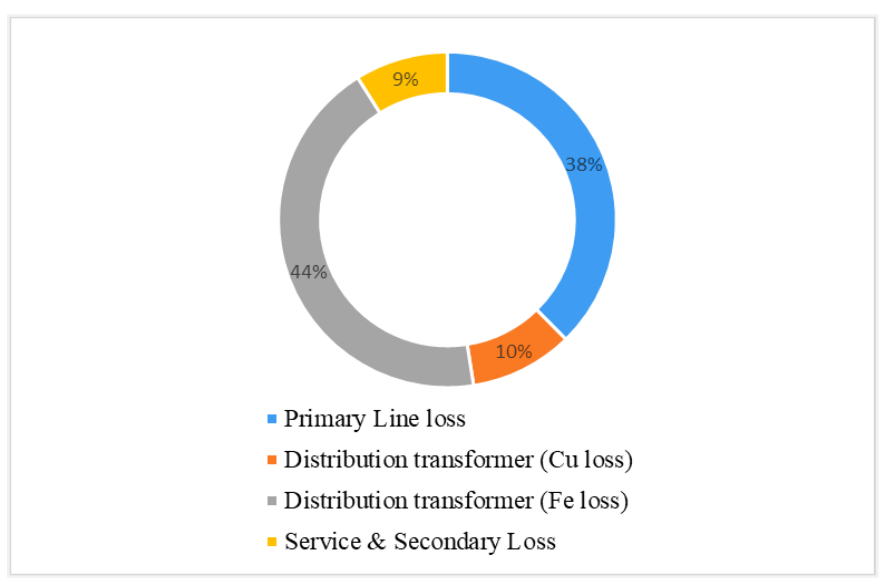

Fig. 1 Various distribution losses estimation, according to EPRI [14].

Hence, it became mandatory to minimize line losses in primary lines at a considerable amount. A different study shows that voltage limits and thermal limits are constrained by higher losses in distribution power systems where maximum loading is limited by mainly voltage limit rather than the thermal limit [15]. To avoid the penalty due to an inferior power factor (p.f.), the SCBs are used. To improve the p.f. three techniques are used, such as centralized compensation, group compensation, and individual compensation. Three different compensation techniques are available in the literature, including individual compensation, group compensation, and centralized compensation to improve the power factor. To get maximum advantages in p.f. correction all the methods, as mentioned earlier, can be used [16]. Synchronous condensers can also be used instead of static SCBs [19] because manufacturers want to produce equipment with improved power factor and higher efficiency [17].

\subsection{The Impact of Reactive Power (QCap) in the Vertically Unbundled Electricity Market.}

Due to the expansion of the electricity market, the unbundled electricity power system is now regulated by Regional Transmission Organizations (RTO) and Independent System Operator (ISO) to assure security, reliability, and quality of the electrical power services. The restructured power market parted as Generation Company (GenCOs), Distribution Company (Discos), and Transmission Company (TransCOs) [18]-[20]. The existing power systems became limited to transmit generated power from central generation to distribution systems due to aging because most of the power systems are more than 40 years old. Hence, these systems unable to cope up 
with growing demands. Besides, transmission investment has reduced at an alarming rate for the last few decades. [21]. Transmission congestion can be relieved by employing FACTS devices, SCBs, Distributed Generations (DGs), voltage regulators, etc., rather than installing new transmission lines [22], [23]. But to supply extra kVAR, it is inevitable to sacrifice real power output. In the real scenario, the utilities prefer to generate more real power for profit maximization [24]. As in the real power market, reactive power is not easy to generate since it does not travel far. Consequently, reactive power has to generate locally [25].

Thus compensation of reactive power ( $\mathrm{Q}$ cap) becomes vital because of deregulation in the power market and conversion of the Network from passive to active. SCBs will be the most cost-effective solution for reactive power compensation because of the lower initial investment, and there is no personnel and maintenance cost. The optimal allocation and sizing of SCBs became a very attractive topic among researchers since nonoptimal sizing and sitting will cost real power losses in distribution feeder as capacitive MVAr and losses have deep bath curve relation [26].

\subsection{Incorporation of DGs in Distribution System and Reactive Power Management by SCBs}

We can call the Distributed Generation (DG) a small-scale generation. It is connected to the distribution level and is a real active power generating unit. Electricity production facilities are necessarily small with respect to central plants, according to IEEE. As a result, it facilitates the interconnection at any close point in the electric power system, as disperse resources. The DGs are considered an electric power generation source connected to the consumer site or the distribution network [27]. They can afford electricity at a cheap price by maintaining higher security and reliability and less environmental pollution than the old-style power generation. In addition, since DGs are not dependent on the main power grid, it can deliver power to a vast number of public services. For instance, educational institutions, airports, hospitals, military bases, police stations, natural gas distribution, transmission systems communication sectors, etc. Virginia Tech's Consortium on Energy Restructuring defines the distribution power network in two categories: the local and endpoint levels. The local generating power plants mostly consists of RE technologies that depend on site such as solar PV systems, WT-DG, geothermal power plant, hydro-thermal generating stations.

On the contrary, at the end-point level, the different customers can apply the same technology. For instance, the modular combustion engine can furnish as home back up and at the same time to other buildings. Hence, disperse generators contribute in a small amount to the main power grid. The main focus of DGs is -friendly to the environment, efficient, and economically viable. These distributed generation based power plants needed reactive power to maintain proper node voltage. Locally generated reactive power from SCBs will be the right choice in this regard.

\subsection{Voltage Profile Improvement (VPI)}

Generally, DGs are treated to supply active power [28]; voltage profile deterioration is a remarkable challenge to the utility due to high DGs penetration at heavy system loading. To maintain a voltage profile at an acceptable limit, certain reactive power always has to be maintained [29]. In the vertically unbundled electricity market, the responsibilities rested on ISO to keep voltage profile in preferable limits by GenCOs. The reactive power supply can be controlled in numerous ways, such as: changing the excitation, by changing tap changing transformers, or by removing reactors and adding capacitive type devices. Voltage control equipment must adhere to DGs because at light load DGs will cause voltage rise problems [30]. Due to environmental pollution and the Greenhouse effect, nonconventional energy resources based on power generation have become popular such as-wind and solar energy. Asynchronous induction generator in case wind power generation must need a local reactive power supply, but this problem can have addressed with Doubly Fed Induction Generator (DFIG). Various reactive power compensation technique has been described in [31] along with SCBs, over-excited synchronous motor, etc. STATCOMs. SVCs and other recent reactive power enhancement devices can be used at the generation level.

\section{Optimum Shunt Capacitor Placement Techniques-A Review}

Different researchers have proposed various formulas and techniques for optimum placement of SCBs considering numerous fitness functions such as power loss minimization, VPI, installation cost reduction, burden reduction on existing lines, maximization of system stability, etc. SCBs are placed in two different ways of fixed and variable (switched) combinations. The variable capacitors' size depends on the difference between existing reactive power demand and available fixed capacitive power. In contrast, fixed capacitors rely on average reactive power needed by the electric power systems. To control the variable SCBs, special control techniques are employed. SCBs $\left(\mathrm{Q}_{\mathrm{Cap}}\right)$ are found in discrete sizes that are multiples of a minimum capacitor size $\mathrm{Q}_{\min }$ that has been given in equation (2) [32], [33]. Both fixed and variable combinations of SCBs are used for continuous sizes. The absolute value of SCBs is achieved by employing a variable capacitor bank.

$Q_{\text {Cap }}=n \times Q_{\min }$

The authors suggested various SCBs sitting problems in different research articles that have been discussed below. Moreover, multidimensional problems also have been addressed in some other research articles considering DGs, reconfiguration of the Network, and voltage regulators. The common algorithm of sitting and sizing of SCBs have demonstrated in Fig. 2.

\subsection{Analytical Methods}

A calculus-based analytical method was proposed at the early stage when suitable computational resources were not available, and computational procedures were reduced by considering approximation. These analytical methods were also had used SCBs sizing and sitting. The work has begun with placing single and multiple capacitors by Neagle in Non-uniform and uniform load conditions. He proposed SCBs to place at 1(1/2) distance from the main substation (SS) [2]. Cook developed a more realistic algorithm considering the average $\mathrm{Q}$ load using fixed SCBs for uniformly distributed load conditions [34]. He proposed that the optimum location of a capacitor bank would be $2 / 3$. The author also extended his work using variable 
SCBs [35]. After that, several analytical methods were also proposed in various literature [36], [37]. Extended research of Cook [34] was done by Schmill [38] with equations for sitting and sizing of $\mathrm{N}$ number of capacitors with a uniformly distributed load on a uniform feeder. The optimal conditions of sitting and sizing for single or double SCBs on a feeder also considered discrete loads and Non-uniform resistance. In this literature, an iterative process had proposed to address the problem. Uniform and a concentrated end load on the distributed feeder was suggested by Chang et al. [39], [40]. Schmill had determined the optimum place of SCB based on the calculation of energy losses and peak power losses, whereas total savings determined the size.

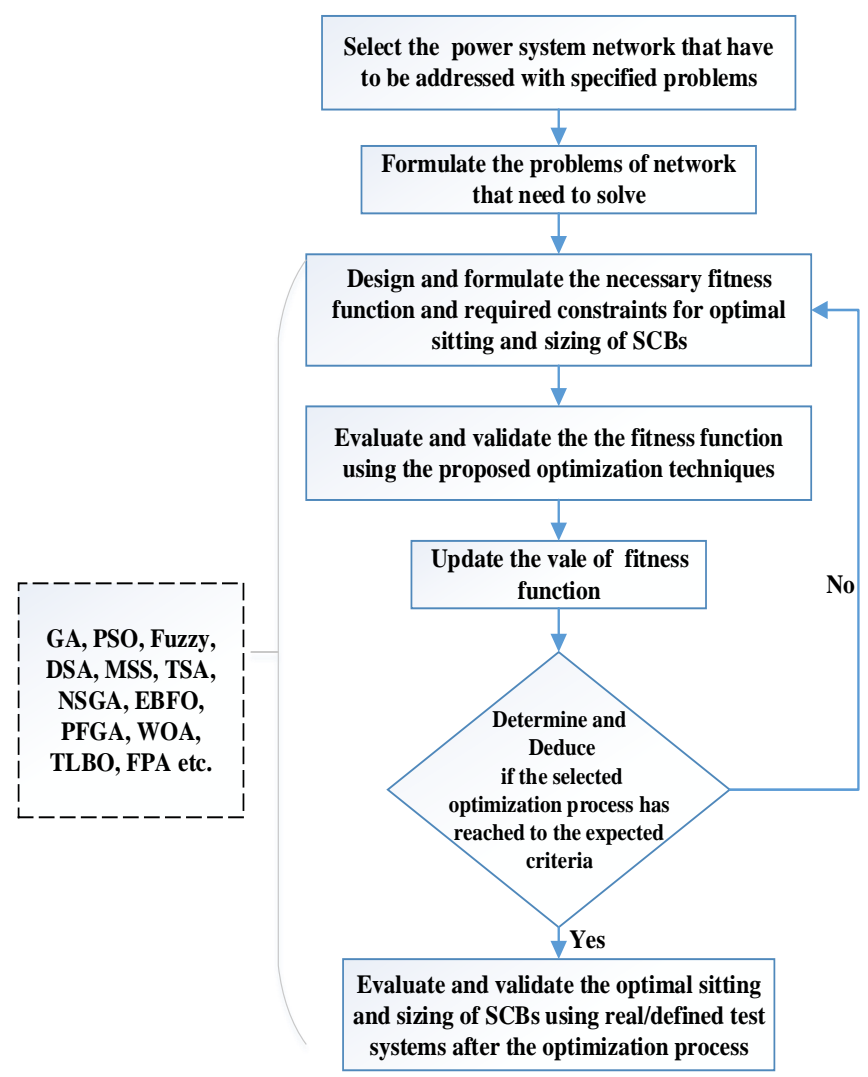

Fig. 2 General Algorithm for sitting and sizing of SCBs.

It is easy for the implementation of an analytical method, and its execution is faster. Since it takes simple presumption and considers one snapshot of the electric power system loading condition. Though Analytical methods are treated as the simplest method, many assumptions and scenarios have to make before finding optimal sizing and sitting of SCBs. The " $2 / 3$ rule" showed negative savings in various scenarios [41]. At the very beginning of the research, sitting and sizing of SCBs, sizes, and locations are considered as continuous variables. Consequently, the calculated results needed to be rounded to the closest real value that may give overvoltage problems or show loss savings in terms of the dollar lower than the calculated values. But recently proposed analytical methods much more convenient, accurate, and practical for distributed systems [37], [42]-[45]. Ardiaty proposed a new formula to measure the Reactive Contribution Index (RCI) of each node. His objective function was to achieve the most stable condition with feeder loss minimization [46]. In article [47], the authors proposed a probabilistic load flow analysis for radial distributions system (PLFRDS) considering stochastic load variations. M. Ihsan et al. developed the Exhaustive method for both real and reactive power reduction [48].

\subsection{Numerical Programming Methods}

In Numerical Programming methods, the mathematical models are formulated and solved arithmetically. It is an iterative process that can minimize or maximize the particular objective function of decision variables with some constraints. The application of Numerical Programming methods has been increased in power systems because of available larger memory chips and fast computation skills [49], [50]. In optimum sizing and sitting of SCBs problems, the researchers suggested various mathematical models and employed Numerical Programming methods to find optimum locations and sizes. The optimal location of SCBs was determined by Duran et al. using dynamic programming and accomplished Schmil work [38] for uniformly and randomly distributed load. The author used discrete capacitors and energy loss reduction, was the objective function [51]. Fawzi et al. extended Duran's work [51] and incorporated the extra kVA as a savings function [52]. The local variations method proposed by Ponnavsikko and Rao used the variable SCBs included the effects of variable load growth [53]. Lee developed an optimization technique that incorporates both fixed and variable SCBs to provide net monetary savings [50]. Baran and $\mathrm{Wu}$ used the mixed-integer programming approach for SCBs placement and sizing [54], [55]. The complete power flow model was used by Sharaf et al. used the full load flow model to find the optimum place of SCBs in a distribution feeder [56]. The author also said that the model developed in [57] is not suitable for optimal placement of SCBs since end-user bus voltage decreased as the system load increased quadratically. Overall energy savings were considered the objective function in the mixedinteger linear problem model proposed by Khodr for SCBs placement problems [58]. In [59], [60], the authors considered Monte Carlo Simulation to deal with stochastic load variations, and the objective function was minimizing was power losses. S. Soto applied the proposed MCS model in a practical subtransmission system [59], and M.B. Jannat applied it in a $35 \mathrm{kV}$ real distribution system [60].

\subsection{Heuristics Methods}

Heuristics methods are called rules of thumb because they are based on suggestions or hints and were developed on experiences, senses, and judgments. These methods minimize the exhaustive search space and furnish almost real and quick decisions and give optimal results with full confidence [61], [62]. Hence, this method-based technique widely applies to optimum SCBs sitting and sizing [63]-[67]. In [63], the authors developed a heuristic method that identified the sensitive node and placed $\mathrm{SCBs}$ to reduce the feeder losses in a significant amount. Chis et al. had elaborated Abdel-Salam et al.'s work considering the cost of SCBs and minimization of energy and peak power loss [64]. The bus bar Sensitivity Index has considered fixing the optimal position and size of SCBs in [65]. Hamouda et al. had used the node voltage stability index to select the optimum location. The objectives function of this research were to maximize the net savings and capacitor investment due to the different size of SCBs [66]. 
Table 1 Summary of Analytical Methods.

\begin{tabular}{|c|c|c|c|c|c|c|c|}
\hline $\begin{array}{l}\text { Publi- } \\
\text { shed }\end{array}$ & Ref. & SCB type & $\begin{array}{l}\text { Design } \\
\text { variables }\end{array}$ & Load profiles & Method & Objective function & Test systems \\
\hline 1956 & {$[2]$} & Fixed & Location & $\begin{array}{l}\text { Uniform and Non- } \\
\text { uniform distributed } \\
\text { load }\end{array}$ & $1-1 / 2 \mathrm{kVA} / \mathrm{kVAr}$ rule & Feeder loss reduction & $\begin{array}{l}\text { Primary } \\
\text { feeders }\end{array}$ \\
\hline 1959 & {$[34]$} & Fixed & Location & Distributed load & $2 / 3$ rule & Feeder loss reduction & $\begin{array}{l}\text { Primary } \\
\text { feeders }\end{array}$ \\
\hline 1961 & {$[35]$} & $\begin{array}{l}\text { Fixed }+ \text { Sw } \\
\text { itched }\end{array}$ & Location & $\begin{array}{l}\text { Uniform distributed } \\
\text { load }\end{array}$ & Energy loss equation & Feeder loss reduction & $\begin{array}{l}\text { Primary } \\
\text { feeders }\end{array}$ \\
\hline 1965 & {$[38]$} & Both & $\begin{array}{l}\text { Location } \\
+ \text { Size }\end{array}$ & $\begin{array}{l}\text { Uniform \& random } \\
\text { distributed }\end{array}$ & Iterative approach & $\begin{array}{l}\text { Feeder active \& reactive } \\
\text { loss reduction }\end{array}$ & $\begin{array}{l}\text { Distribution } \\
\text { feeder }\end{array}$ \\
\hline 1969 & [39] & Switched & $\begin{array}{l}\text { Location } \\
+ \text { Size }\end{array}$ & Uniform load & $\begin{array}{l}\text { Computer-based new } \\
\text { Iterative approach }\end{array}$ & $\begin{array}{l}\text { Optimization of total } \\
\text { monetary savings }\end{array}$ & $\begin{array}{l}\text { Primary } \\
\text { feeders }\end{array}$ \\
\hline 1972 & {$[40]$} & Switched & $\begin{array}{l}\text { Location } \\
+ \text { Size }\end{array}$ & $\begin{array}{l}\text { Concentrated and } \\
\text { uniformly distributed } \\
\text { load }\end{array}$ & $\begin{array}{l}\text { Determining generalized } \\
\text { loss equation }\end{array}$ & Economic savings & $\begin{array}{l}\text { Distribution } \\
\text { feeder }\end{array}$ \\
\hline 1978 & {$[36]$} & Fixed & Location & $\begin{array}{l}\text { Uniform distributed } \\
\text { load }\end{array}$ & General loss equation & Yearly loss reduction & $\begin{array}{l}\text { Distribution } \\
\text { feeder }\end{array}$ \\
\hline 1981 & {$[37]$} & Both & $\begin{array}{l}\text { Location } \\
+ \text { Size }\end{array}$ & $\begin{array}{l}\text { Uniform distributed } \\
\text { load }\end{array}$ & Equal area criterion & Loss reduction & $\begin{array}{l}\text { Distribution } \\
\text { feeder }\end{array}$ \\
\hline 1985 & [43] & Switched & $\begin{array}{l}\text { Location } \\
+ \text { Size }\end{array}$ & $\begin{array}{l}\text { Varying load } \\
\text { condition }\end{array}$ & Step by step calculation & $\begin{array}{l}\text { Peak power loss and } \\
\text { energy loss reduction }\end{array}$ & $\begin{array}{l}\text { Distribution } \\
\text { feeder }\end{array}$ \\
\hline 1985 & {$[42]$} & Switched & $\begin{array}{l}\text { Location } \\
+ \text { Size }\end{array}$ & $\begin{array}{l}\text { Uniform feeder with } \\
\text { an end-load }\end{array}$ & General loss equation & $\begin{array}{l}\text { Peak power loss and } \\
\text { energy loss reduction }\end{array}$ & $\begin{array}{l}\text { Distribution } \\
\text { feeder }\end{array}$ \\
\hline 1997 & {$[45]$} & Both & $\begin{array}{l}\text { Location } \\
+ \text { Size }\end{array}$ & Time-varying load & Three-phases load flow & Minimizing the loss & $\begin{array}{l}\text { Taiwan LY- } \\
37, \mathrm{BX} 33 \\
\end{array}$ \\
\hline 1999 & [44] & Switched & $\begin{array}{l}\text { Location } \\
+ \text { Size }\end{array}$ & Time-varying load & Iterative approach & Significant loss savings & $\begin{array}{l}\text { 15-bus \& } \\
\text { 33-bus }\end{array}$ \\
\hline 2009 & [156] & Fixed & $\begin{array}{l}\text { Location } \\
+ \text { Size }\end{array}$ & Fixed load & $\begin{array}{l}\text { BIBC \& BCBV based } \\
\text { new method }\end{array}$ & Minimizing power loss & $\begin{array}{l}12,34,69- \\
\text { bus }\end{array}$ \\
\hline 2016 & [46] & Fixed & Location & Non-uniform load & $\begin{array}{l}\text { Improved Modal Analysis } \\
\text { with RCI }\end{array}$ & $\begin{array}{l}\text { Achieve stable condition } \\
\& \text { Minimize power loss }\end{array}$ & IEEE 30-bus \\
\hline 2019 & {$[47]$} & Fixed & $\begin{array}{l}\text { Location } \\
+ \text { Size }\end{array}$ & $\begin{array}{l}\text { Stochastic Load } \\
\text { variation }\end{array}$ & PLFRDS method & $\begin{array}{l}\text { Loss reduction \& } \\
\text { improve Voltage profile }\end{array}$ & 30, 85-bus \\
\hline 2019 & [48] & Fixed & $\begin{array}{l}\text { Location } \\
+ \text { Size }\end{array}$ & Average load & $\begin{array}{l}\text { Analytical expression \& } \\
\text { exhaustive method }\end{array}$ & $\begin{array}{l}\text { P \& Q Loss reduction \& } \\
\text { improve Voltage profile }\end{array}$ & IEEE 37-bus \\
\hline
\end{tabular}

Table 2 Summary of Numerical Programming Methods.

\begin{tabular}{|c|c|c|c|c|c|c|c|}
\hline $\begin{array}{l}\begin{array}{l}\text { Publi- } \\
\text { shed }\end{array} \\
\end{array}$ & Ref. & SCB type & \begin{tabular}{|l|} 
Design \\
variables
\end{tabular} & Load profiles & Method & Objective function & Test systems \\
\hline 1968 & {$[51]$} & Switched & $\begin{array}{l}\begin{array}{l}\text { Location } \\
+ \text { Size }\end{array} \\
\end{array}$ & Discrete lumped loads & Dynamic programming & Minimize the power loss & Distribution feeder \\
\hline 1981 & {$[37]$} & Both & $\begin{array}{l}\text { Location } \\
+ \text { Size }\end{array}$ & Non-uniform load & Iterative technique & Net monetary savings & $\begin{array}{l}\text { A certain point on } \\
\text { feeder }\end{array}$ \\
\hline 1983 & {$[52]$} & Switched & Location & $\begin{array}{l}\text { Uniform \& random } \\
\text { distributed load }\end{array}$ & Dynamic programming & Minimize the power loss & $\begin{array}{l}\text { Rural Distribution, } \\
\text { Egypt }\end{array}$ \\
\hline 1983 & {$[53]$} & Both & $\begin{array}{l}\text { Location } \\
+ \text { Size }\end{array}$ & $\begin{array}{l}\text { Load growth with } \\
\text { varying load }\end{array}$ & Local variation method & Minimize the power loss & $\begin{array}{l}\text { Indian Distribution } \\
\text { feeder }\end{array}$ \\
\hline 1989 & {$[54]$} & Both & Size & Time-varying load & non-linear programming & $\begin{array}{l}\text { Power loss minimization } \\
\text { \& Voltage regulation }\end{array}$ & Distribution feeder \\
\hline 1989 & {$[55]$} & Switched & $\begin{array}{l}\text { Location } \\
+ \text { Size }\end{array}$ & $\begin{array}{l}\text { Uniform concentrated } \\
\text { end load }\end{array}$ & Mixed-integer program & $\begin{array}{l}\text { Peak power loss and } \\
\text { energy loss reduction }\end{array}$ & $\begin{array}{l}\text { TS1, TS2 } \\
\text { Distribution feeder }\end{array}$ \\
\hline 1996 & {$[56]$} & Switched & $\begin{array}{l}\text { Location } \\
+ \text { Size }\end{array}$ & Distributed load & FLFM, EGSLM model & Cost minimization & 18-bus system \\
\hline 2008 & {$[58]$} & Switched & $\begin{array}{l}\text { Location } \\
+ \text { Size }\end{array}$ & Single load level & $\begin{array}{l}\text { mixed-integer linear } \\
\text { problem }\end{array}$ & Minimize the power loss & 15-bus, 33 bus test \\
\hline 2016 & [59] & Fixed & Location & Stochastic Load & MCS & Power loss minimization & $\begin{array}{l}\text { Real sub- } \\
\text { transmission system }\end{array}$ \\
\hline 2016 & {$[60]$} & Fixed & Location & Random Load & MCS & $\begin{array}{l}\text { Active energy loss } \\
\text { minimization }\end{array}$ & $\begin{array}{l}35 \mathrm{kV} \text { real } \\
\text { distribution network }\end{array}$ \\
\hline
\end{tabular}


To fix the optimum place of SCBs, the weakest line has taken as candidate bus, and the optimum size was selected by using PSO that gave minimum feeder losses. Raju et al. proposed the DSA algorithm that assures net savings maximization and voltage profile improvement. The optimal size and location of fixed and variable SCBs were determined in the radial feeder by applying the DSA algorithm [67]. To determine location and size, both fixed and variable SCBs have been used in articles [68]-[71]. Accelerated PSO has been used to reduce net benefits, and Cuckoo Search Algorithm (CSA) has been used to minify system operating \& improve voltage profiles at different load levels [67], [68]. SSO algorithm used for Cost minimization due to energy loss \& reactive power compensation [70] and Modified Gbest-guided Artificial Bee Colony (MGABC) algorithm has applied for minimization of power loss, total annual expense and voltage deviation [70] in 34 \& 118-bus distribution systems. Researchers also proposed numerous SCBs algorithms and methods such as the HCODECQ method [72], BFOA method [73], Crow Search Algorithm (CSA) [74], HSA-PABC algorithm [75]. A. Mujezinović et al. developed a Load flow calculation algorithm and integer genetic algorithm on a $10 \mathrm{kV}$ distribution network in Bosnia \& Herzegovina that reduce power losses and improve bus voltages [76].

Table 3 Summary of Heuristics Methods.

\begin{tabular}{|c|c|c|c|c|c|c|c|}
\hline \begin{tabular}{|l|} 
Publ- \\
ished
\end{tabular} & Ref. & SCB type & \begin{tabular}{|l|} 
Design \\
variables
\end{tabular} & Load profiles & Method & Objective function & Test systems \\
\hline 1994 & {$[63]$} & Fixed & Size & Variable load & $\begin{array}{l}\text { New loss reduction } \\
\text { technique }\end{array}$ & Minimize reactive loss & 45-bus \\
\hline 1997 & {$[64]$} & Fixed & $\begin{array}{l}\text { Location } \\
+ \text { Size }\end{array}$ & Average load & $\begin{array}{l}\text { Sensitive node } \\
\text { searching }\end{array}$ & $\begin{array}{l}\text { Minimize the power } \\
\text { loss }\end{array}$ & 34-bus \\
\hline 2008 & {$[65]$} & Fixed & Location & Different load conditions & HCA algorithm & Net annual savings & 70, 476-bus \\
\hline 2012 & {$[26]$} & Both & $\begin{array}{l}\text { Location } \\
+ \text { Size }\end{array}$ & Different load conditions & $\begin{array}{l}\text { RVSI V Indexing } \\
\text { method }\end{array}$ & $\begin{array}{l}\text { Minimize the power } \\
\text { loss }\end{array}$ & $12,33,69$-bus \\
\hline 2012 & {$[67]$} & Both & $\begin{array}{l}\text { Location } \\
+ \text { Size }\end{array}$ & Average load & $\begin{array}{l}\text { Direct Search } \\
\text { Algorithm }\end{array}$ & $\begin{array}{l}\text { Net savings and } \\
\text { improve voltage } \\
\text { profiles }\end{array}$ & $22,69,85$-bus \\
\hline 2013 & {$[66]$} & Both & $\begin{array}{l}\text { Location } \\
+ \text { Size }\end{array}$ & Average load & $\begin{array}{l}\text { Heuristic search } \\
\text { method }\end{array}$ & $\begin{array}{l}\text { Net savings and } \\
\text { improve voltage } \\
\text { profiles }\end{array}$ & $10,22,69$-bus \\
\hline 2014 & {$[68]$} & Both & $\begin{array}{l}\text { Location } \\
+ \text { Size }\end{array}$ & Different load conditions & Accelerated PSO & Maximize net benefits & 34 \& 118-bus \\
\hline 2014 & [69] & Both & $\begin{array}{l}\text { Location } \\
+ \text { Size }\end{array}$ & Different loading levels & $\begin{array}{l}\text { Cuckoo Search } \\
\text { Algorithm }\end{array}$ & $\begin{array}{l}\text { Minify system } \\
\text { operating \& improve } \\
\text { voltage profiles }\end{array}$ & 69 \& 118-bus \\
\hline 2015 & {$[70]$} & Fixed & Location & Average load & HCODECQ method & $\begin{array}{l}\text { Power loss } \\
\text { minimization }\end{array}$ & $33,66,132$-bus \\
\hline 2015 & {$[73]$} & Switched & $\begin{array}{l}\text { Location } \\
+ \text { Size }\end{array}$ & Different loading levels & BFOA method & $\begin{array}{l}\text { Minimize the power } \\
\text { loss }\end{array}$ & $34 \& 85$-bus \\
\hline 2016 & {$[74]$} & Fixed & Location & Different load conditions & $\begin{array}{l}\text { Crow Search } \\
\text { Algorithm }\end{array}$ & $\begin{array}{l}\text { Minimize power losses } \\
\text { and improve voltage } \\
\text { profiles }\end{array}$ & $9 \& 33$-bus \\
\hline 2016 & {$[70]$} & Both & $\begin{array}{l}\text { Location } \\
+ \text { Size }\end{array}$ & Average load & $\begin{array}{l}\text { Shark Smell } \\
\text { Optimization (SSO) } \\
\text { algorithm }\end{array}$ & $\begin{array}{l}\text { Cost minimization due } \\
\text { to energy loss \& } \\
\text { reactive power } \\
\text { compensation }\end{array}$ & 34 \& 118-bus \\
\hline 2018 & [71] & Both & $\begin{array}{l}\text { Location } \\
+ \text { Size }\end{array}$ & Various load levels & MGABC algorithm & $\begin{array}{l}\text { minimization of power } \\
\text { loss, total annual } \\
\text { expense, and voltage } \\
\text { deviation }\end{array}$ & 34,118 -bus \\
\hline 2018 & [75] & Switched & $\begin{array}{l}\text { Location } \\
+ \text { Size }\end{array}$ & $\begin{array}{l}\text { Voltage-dependent load } \\
\text { models }\end{array}$ & $\begin{array}{l}\text { HSA-PABC } \\
\text { algorithm }\end{array}$ & $\begin{array}{l}\text { Power loss reduction, } \\
\text { voltage stability } \\
\text { improvement, and net } \\
\text { annual savings }\end{array}$ & 69,118 -bus \\
\hline 2019 & {$[76]$} & Fixed & $\begin{array}{l}\text { Location } \\
+ \text { Size }\end{array}$ & Average load & $\begin{array}{l}\text { Load flow } \\
\text { calculation algorithm } \\
\text { \& integer genetic } \\
\text { algorithm }\end{array}$ & $\begin{array}{l}\text { Minimize power losses } \\
\text { and improve voltage } \\
\text { profiles }\end{array}$ & $\begin{array}{l}10 \mathrm{kV} \text { dist. real } \\
\text { Network in } \\
\text { Bosnia }\end{array}$ \\
\hline
\end{tabular}




\subsection{Artificial Intelligent Methods}

Exhaustive search is the simplest search algorithm in the optimization technique since it searches all probable solutions from a set of predefined values. But this method is considered an inefficient technique because it needed higher computational time and space. Kokash proposed a new special class of heuristic techniques based on nature, intelligence, and greedy known as the Artificial Intelligent (AI) method [77]. This AI method has been employed to find the optimal place and size of SCBs on distribution systems. Many researchers use AI methods as one of the most potent methods to solve power system problems, but it is needed higher computation time and memory space [78]. Different researcher has been proposed various algorithm such as: GA [79]-[84], Fuzzy [85], [86], Fuzzy-GA [87]-[88], Particle
Swarm Optimization (PSO) [89]-[90], Immune Algorithm (IA) [91], Plant Growth Simulation Algorithm (PGSA) [92], Tabu Search (TS) [93], Memetic-Algorithm Approach [94], TLBO algorithm [95], Ant Colony [96], Graph Search Algorithm (GSA) [97]-[98], Artificial Bee Colony (ABC) [99], and Hybrid Algorithm [100]-[102]. The authors proposed CSA Optimization [68], a new algorithm of Inclusion and interchange of variables [103], Flower Pollination Algorithm [104] in the various distribution network to minify total cost. Moreover, to improve net savings and bus voltage, the researcher suggested different methods to connect fixed and switched SCBs that given as Fuzzy-Real Coded GA algorithm [105], BA and CS method [106], Loss sensitivity approach [107], GAs and SA analysis [108], PSO and Improved BSFS [109], WOA Algorithm [110].

Table 4 Summary of Artificial intelligent Methods.

\begin{tabular}{|c|c|c|c|c|c|c|c|}
\hline $\begin{array}{l}\text { Publi- } \\
\text { shed }\end{array}$ & Ref. & $\begin{array}{l}\mathrm{SCB} \\
\text { type }\end{array}$ & \begin{tabular}{|l} 
Design \\
variables
\end{tabular} & Load profiles & Method & Objective function & Test systems \\
\hline 1990 & [113] & Fixed & Size & $\begin{array}{l}\text { Linear and time- } \\
\text { invariant load }\end{array}$ & Numerical algorithm & $\begin{array}{l}\text { Reduction of total power } \\
\text { loss \& THD }\end{array}$ & $\begin{array}{l}\text { Radial Dist. } \\
\text { Feeder }\end{array}$ \\
\hline 1993 & [83] & Fixed & Location & $\begin{array}{l}\text { Differential load } \\
\text { pattern }\end{array}$ & GA method & Minimize the power loss & 69-bus \\
\hline 1994 & [79] & Both & $\begin{array}{l}\text { Location } \\
+ \text { Size }\end{array}$ & Average load & GA method & Minimize the power loss & 9,30-bus \\
\hline 1995 & [114] & Both & Size & Different load levels & MSS method & $\begin{array}{l}\text { Cost and substation } \\
\text { Harmonic reduction }\end{array}$ & $\begin{array}{l}23 \mathrm{kV} \\
\text { distributor }\end{array}$ \\
\hline 1999 & [102] & Fixed & $\begin{array}{l}\text { Location } \\
+ \text { Size }\end{array}$ & Various load levels & Basic search technique & Minimize system cost & $\begin{array}{l}\text { Distribution } \\
\text { feeder }\end{array}$ \\
\hline 2000 & {$[82]$} & Fixed & Location & $\begin{array}{l}\text { Differential load } \\
\text { pattern }\end{array}$ & $\begin{array}{l}\text { GA \& Fast energy loss } \\
\text { reduction technique }\end{array}$ & $\begin{array}{l}\text { Overall power and energy } \\
\text { loss minimization }\end{array}$ & $\begin{array}{l}\text { Single feeder } \\
\text { fed by } 24 \mathrm{kV}, \\
15 \mathrm{MVA}\end{array}$ \\
\hline 2000 & {$[85]$} & Fixed & Size & Average load & $\begin{array}{l}\text { Approximate } \\
\text { reasoning with FES }\end{array}$ & Net energy savings & 34-bus \\
\hline 2000 & [91] & Fixed & Location & Different load levels & IA based optimization & Minimize power loss & 69-bus \\
\hline 2000 & {$[97]$} & Both & $\begin{array}{l}\text { Location } \\
+ \text { Size }\end{array}$ & Average load & $\begin{array}{l}\text { Graph search } \\
\text { algorithm }\end{array}$ & Overall savings & Practical feeder \\
\hline 2001 & {$[100]$} & Fixed & $\begin{array}{l}\text { Location } \\
+ \text { Size }\end{array}$ & Different load levels & Hybrid method & Cost savings & $9,65,135$-bus \\
\hline 2001 & [132] & Switched & Location & Different load levels & $\begin{array}{l}\text { Simulated annealing } \\
\text { technique }\end{array}$ & $\begin{array}{l}\text { Minimize power loss and } \\
\text { improve voltage profiles }\end{array}$ & $\begin{array}{l}\text { IEEE 3-feeder } \\
\text { system }\end{array}$ \\
\hline 2002 & {$[80]$} & Both & Size & Varying load & GA method & Minimize reactive loss & 69-bus \\
\hline 2002 & {$[117]$} & Both & $\begin{array}{l}\text { Location } \\
+ \text { Size }\end{array}$ & $\begin{array}{l}\text { Linear and } \\
\text { nonlinear loads }\end{array}$ & $\begin{array}{l}\text { HARMFLOW } \\
\text { algorithm and MSS } \\
\text { method }\end{array}$ & $\begin{array}{l}\text { Minimize system losses } \\
\text { and capacitor cost }\end{array}$ & $\begin{array}{l}\text { 18-Bus IEEE } \\
\text { Distorted } \\
\text { System } \\
\end{array}$ \\
\hline 2004 & [111] & Fixed & Location & Different load levels & NSGA method & $\begin{array}{l}\text { Power loss reduction, p.f. } \\
\text { correction }\end{array}$ & $\begin{array}{l}\text { Distribution } \\
\text { feeder }\end{array}$ \\
\hline 2004 & [115] & Switched & $\begin{array}{l}\text { Location } \\
+ \text { Size }\end{array}$ & Different load levels & PSO algorithm & $\begin{array}{l}\text { Minimize capacitor cost, } \\
\text { energy \& power loss }\end{array}$ & IEEE 9-bus \\
\hline 2004 & {$[116]$} & Fixed & Location & Average load & New GA approach & $\begin{array}{l}\text { Minimize energy, power } \\
\text { loss, and capacitor cost }\end{array}$ & $\begin{array}{l}6 \text { \& 18-Bus } \\
\text { IEEE Distorted } \\
\text { System }\end{array}$ \\
\hline 2004 & [118] & Both & $\begin{array}{l}\text { Location } \\
+ \text { Size }\end{array}$ & Different load levels & MSS-LV optimization & $\begin{array}{l}\text { Minimize capacitor cost, } \\
\text { energy \& power loss }\end{array}$ & $\begin{array}{l}\text { IEEE 18-bus } \\
\text { distorted } \\
\text { System } \\
\end{array}$ \\
\hline 2004 & {$[119]$} & Fixed & $\begin{array}{l}\text { Location } \\
+ \text { Size }\end{array}$ & $\begin{array}{l}\text { Linear and } \\
\text { nonlinear loads }\end{array}$ & $\begin{array}{l}\text { Fuzzy based } \\
\text { approach }\end{array}$ & $\begin{array}{l}\text { Minimize system losses } \\
\text { and capacitor cost }\end{array}$ & $\begin{array}{l}\text { 18-Bus IEEE } \\
\text { Distorted } \\
\text { System }\end{array}$ \\
\hline 2005 & [93] & Fixed & Location & Different load levels & Tabu Search approach & $\begin{array}{l}\text { Minimize power loss and } \\
\text { capacitor cost }\end{array}$ & $\begin{array}{l}\text { 94-bus practical } \\
\text { system }\end{array}$ \\
\hline
\end{tabular}


S. M. G. Mostafa et. al. /JEA Vol. 01(04) 2020, pp 150-169

\begin{tabular}{|c|c|c|c|c|c|c|c|}
\hline $\begin{array}{l}\text { Publi- } \\
\text { shed }\end{array}$ & Ref. & \begin{tabular}{|l}
$\mathrm{SCB}$ \\
type
\end{tabular} & \begin{tabular}{|l} 
Design \\
variables
\end{tabular} & Load profiles & Method & Objective function & Test systems \\
\hline 2005 & [94] & Fixed & $\begin{array}{l}\text { Location } \\
+ \text { Size }\end{array}$ & Average load & $\begin{array}{l}\text { Evolutionary } \\
\text { algorithms }\end{array}$ & Annual cost savings & 9,69-bus \\
\hline 2007 & [81] & Both & $\begin{array}{l}\text { Location } \\
+ \text { Size }\end{array}$ & $\begin{array}{l}\text { Uncertain and time } \\
\text { varying loads }\end{array}$ & $\begin{array}{l}\text { GA method with new } \\
\text { coding }\end{array}$ & $\begin{array}{l}\text { Minimize power loss and } \\
\text { improve voltage profiles }\end{array}$ & $\begin{array}{l}\text { 37,69-bus, a real } \\
\text { Iranian network }\end{array}$ \\
\hline 2007 & [87] & Both & $\begin{array}{l}\text { Location } \\
+ \text { Size }\end{array}$ & Different load levels & Fuzzy-GA method & $\begin{array}{l}\text { Net savings and improve } \\
\text { voltage profiles }\end{array}$ & 69-bus \\
\hline 2009 & [86] & Fixed & $\begin{array}{l}\text { Location } \\
+ \text { Size }\end{array}$ & $\begin{array}{l}\text { Different loading } \\
\text { conditions }\end{array}$ & $\begin{array}{l}\text { A fuzzy based new } \\
\text { method }\end{array}$ & $\begin{array}{l}\text { Minimize power loss and } \\
\text { improve voltage profiles }\end{array}$ & $10,23,34$-bus \\
\hline 2011 & [101] & Switched & Size & Average load & $\begin{array}{l}\text { Fuzzy-DE, Fuzzy- } \\
\text { MAPSO methods }\end{array}$ & $\begin{array}{l}\text { Minimize power loss and } \\
\text { improve voltage profiles }\end{array}$ & 15,34-bus \\
\hline 2012 & [89] & Both & $\begin{array}{l}\text { Location } \\
+ \text { Size }\end{array}$ & Different load levels & $\begin{array}{l}\text { PSO static and } \\
\text { dynamic sensitivity }\end{array}$ & $\begin{array}{l}\text { Minimize capacitor cost } \\
\text { function \& energy loss }\end{array}$ & $70 \& 135$-bus \\
\hline 2012 & [92] & Both & $\begin{array}{l}\text { Location } \\
+ \text { Size }\end{array}$ & Different load levels & $\begin{array}{l}\text { Plant Growth-Based } \\
\text { Optimization }\end{array}$ & $\begin{array}{l}\text { Emission decrement \& } \\
\text { power loss improvement }\end{array}$ & $\begin{array}{l}69,123 \& 17- \\
\text { bus Taipower } \\
\text { company }\end{array}$ \\
\hline 2013 & {$[96]$} & Fixed & $\begin{array}{l}\text { Location } \\
+ \text { Size }\end{array}$ & Load growth model & $\begin{array}{l}\text { Multi period dynamic } \\
\text { model }\end{array}$ & Minimizing the total & 69-bus \\
\hline 2014 & {$[95]$} & Both & $\begin{array}{l}\text { Location } \\
+ \text { Size }\end{array}$ & Different load levels & TLBO approach & $\begin{array}{l}\text { Minimize power loss and } \\
\text { energy cost }\end{array}$ & $\begin{array}{l}22,69,85 \& \\
141 \text {-bus }\end{array}$ \\
\hline 2014 & {$[68]$} & Both & $\begin{array}{l}\text { Location } \\
+ \text { Size }\end{array}$ & $\begin{array}{l}\text { Different loading } \\
\text { conditions }\end{array}$ & CSA Optimization & Minify operating cost & 69 \& 118-bus \\
\hline 2014 & [69] & Both & $\begin{array}{l}\text { Location } \\
+ \text { Size }\end{array}$ & Different load levels & Fuzzy-Real Coded GA & $\begin{array}{l}\text { enhance voltage stability \& } \\
\text { Net savings }\end{array}$ & 33-bus \\
\hline 2015 & {$[84]$} & Fixed & $\begin{array}{l}\text { Location } \\
+ \text { Size }\end{array}$ & Average load & GA & $\begin{array}{l}\text { Improve voltage profiles \& } \\
\text { Minimize power loss }\end{array}$ & 34-bus \\
\hline 2015 & [106] & Both & $\begin{array}{l}\text { Location } \\
+ \text { Size }\end{array}$ & Different load levels & BA and CS method & $\begin{array}{l}\text { Minimize power loss \& } \\
\text { maximize network savings }\end{array}$ & 34, 85-bus \\
\hline 2015 & [107] & Switched & $\begin{array}{l}\text { Location } \\
+ \text { Size }\end{array}$ & $\begin{array}{l}\text { Time varying ZIP } \\
\text { loads }\end{array}$ & $\begin{array}{l}\text { Loss sensitivity } \\
\text { approach }\end{array}$ & $\begin{array}{l}\text { Minimize power loss and } \\
\text { improve voltage profiles }\end{array}$ & $\begin{array}{l}\text { 38-bus UK } \\
\text { distribution } \\
\text { System }\end{array}$ \\
\hline 2015 & {$[90]$} & Fixed & Location & Different load levels & PSO method & $\begin{array}{l}\text { Reduce peak power loss } \\
\text { and improve node voltage }\end{array}$ & 69-bus \\
\hline 2015 & [98] & Fixed & $\begin{array}{l}\text { Location } \\
+ \text { Size }\end{array}$ & Average load & GSA method & $\begin{array}{l}\text { Minimize kW loss and } \\
\text { maximize net savings }\end{array}$ & $\begin{array}{l}33,69,85,141- \\
\text { bus }\end{array}$ \\
\hline 2016 & [108] & Fixed & Location & Average load & GAs and SA analysis & $\begin{array}{l}\text { Minimize power loss and } \\
\text { improve voltage profiles }\end{array}$ & 34, 70-bus \\
\hline 2016 & [109] & Switched & Location & Different load levels & $\begin{array}{l}\text { PSO and Improved } \\
\text { BSFS }\end{array}$ & $\begin{array}{l}\text { Maximize the net annual } \\
\text { returns }\end{array}$ & $\begin{array}{l}\text { A real } \\
\text { unbalanced MV } \\
\text { network }\end{array}$ \\
\hline 2016 & [88] & Both & $\begin{array}{l}\text { Location } \\
+ \text { Size }\end{array}$ & Various load levels & Fuzzy GA Method & $\begin{array}{l}\text { Improve the substation } \\
\text { power factor }\end{array}$ & 51, 69-bus \\
\hline 2016 & [121] & Switched & $\begin{array}{l}\text { Location } \\
+ \text { Size }\end{array}$ & $\begin{array}{l}\text { Future load and } \\
\text { contingency }\end{array}$ & EBFO Method & $\begin{array}{l}\text { Thermal re-rating of } \\
\text { critical cables }\end{array}$ & $\begin{array}{l}\text { Real-world } 110 \\
\text { kV sub-trans. } \\
\text { net. }\end{array}$ \\
\hline 2016 & [122] & Fixed & $\begin{array}{l}\text { Location } \\
+ \text { Size }\end{array}$ & $\begin{array}{l}\text { Different load } \\
\text { models }\end{array}$ & PFGA algorithm & $\begin{array}{l}\text { Cost reduction \& power } \\
\text { quality improvement }\end{array}$ & $18,69,141$-bus \\
\hline 2017 & {$[120]$} & Both & $\begin{array}{l}\text { Location } \\
+ \text { Size } \\
\end{array}$ & Different load levels & MSPSO algorithm & $\begin{array}{l}\text { Maximize net savings, } \\
\text { THD of voltage }\end{array}$ & 18, 69-bus \\
\hline 2017 & [110] & Fixed & $\begin{array}{l}\text { Location } \\
+ \text { Size }\end{array}$ & Average load & WOA Algorithm & $\begin{array}{l}\text { Operating cost and power } \\
\text { loss minimization }\end{array}$ & 34, 85-bus \\
\hline 2017 & [103] & Both & $\begin{array}{l}\text { Location } \\
+ \text { Size }\end{array}$ & Different load states & $\begin{array}{l}\text { Algorithm of Inclusion } \\
\text { and interchange of } \\
\text { variables }\end{array}$ & $\begin{array}{l}\text { Minimize the annual total } \\
\text { cost }\end{array}$ & 69-bus \\
\hline 2017 & [112] & Fixed & $\begin{array}{l}\text { Location } \\
+ \text { Size }\end{array}$ & Average load & NSGA II & $\begin{array}{l}\text { power loss and the THD } \\
\text { minimization }\end{array}$ & 9,85 -bus \\
\hline 2018 & [104] & Fixed & $\begin{array}{l}\text { Location } \\
+ \text { Size }\end{array}$ & Average load & $\begin{array}{l}\text { Flower Pollination } \\
\text { Algorithm (FPA) }\end{array}$ & $\begin{array}{l}\text { Minimize the total power } \\
\text { loss and cost of capacitor } \\
\text { installation }\end{array}$ & $\begin{array}{l}33,34,69,85- \\
\text { bus }\end{array}$ \\
\hline
\end{tabular}


A multi-criteria SCBs placement problem had proposed using the Non-dominated Sorting Genetic Algorithm (NSGA) in [111]. It is needed to optimize the number of objectives simultaneously in NSGA. Moreover, in NSGA, any objective can be optimized without deterioration of other objective functions. So, Pareto-Optimal solutions are considered to fulfill the objective function [10]. Baghzouz and $\mathrm{Wu}$ had developed a method to optimize the size of SCBs in radial distribution feeder considering r.m.s. voltage and their corresponding total harmonic distortion. NSGS-II was introduced in [112] to reduce power losses and ensure the THD maintains power quality. The authors found that the optimal sizing of SCBs will cause unexpected distortion in voltage profiles when harmonic distortion is neglected [113]-[114]. The researcher used the PSO algorithm for finding the optimum size, location, and type considering non-linear loads in [115]. Yu et al. applied the GA algorithm to address the SCBs placement and sizing problem by incorporating the impact of voltage and current harmonics [116]. The researcher had demanded that the applied method minimized THD and confirm higher annual benefits in contrast with [117][119]. MSPSO algorithm was applied in [120] where the fitness function was a net yearly benefit, maximum THD of voltage, maximum voltage deviation, and a resonance constraint. A.M. Othman developed the EBFO technique that incorporates optimal SCBs sizing and sitting with thermal cable evaluation on a practical $110 \mathrm{kV}$ sub-transmission line [121]. PFGA algorithm has provided Cost reduction \& power quality improvement in radial distribution systems [122].

\subsection{Multi-dimensional Problems}

In some research articles, authors considered other power system problems with SCBs such as- Placement of Distributed Generations (DGs) [123]-[131], reconfiguration of the Network [132]-[140], load tap changer [141], placement of voltage regulators [142]-[148], etc. Voltage regulator and SCBs placement performed simultaneously to control voltage and var [142]-[144]. Hung et al. proposed a multidimensional algorithm that associated SCBs, DGs, and network reconfiguration in a single objective function to reduce distribution feeder losses significantly [149]. Adel et al. proposed a Water Cycle Algorithm (WCA) to size and sit of SCBs and DGs that reduce power losses, voltage deviation, electrical energy cost, and total emissions [150]. WCA was also incorporated in the article [151], where the authors suggested two load power factor models to minify feeder losses and voltage profile enhancement. GA interfaced with COM model has developed for optimal phase reconfiguration and SCBs placement [152]. In [153], a Hybrid WIPSO-GSA algorithm has been proposed in distribution systems considering feeder failure rate. Feeders reconfiguration and SCBs placement done by Mixed-integer second-order cone programming model [154]. The authors proposed a methodology for the sustainable operation of distribution systems along with sitting and sizing of SCBs and dispatchable DGs. Sensitivity analysis based on voltage stability index has been employed to minimize feeder current, power loss, and improve voltage profiles [155].

Table 5 Summary of Multi-dimensional problems.

\begin{tabular}{|c|c|c|c|c|c|c|c|}
\hline $\begin{array}{l}\text { Publi- } \\
\text { shed }\end{array}$ & Ref. & $\begin{array}{l}\text { SCB } \\
\text { type }\end{array}$ & \begin{tabular}{|l} 
Design \\
variables
\end{tabular} & Load profiles & Method & Objective function & Test systems \\
\hline 1985 & [142] & Both & $\begin{array}{l}\text { Location } \\
+ \text { Size }\end{array}$ & $\begin{array}{l}\text { Variable load } \\
\text { conditions }\end{array}$ & Analytical Method & $\begin{array}{l}\text { Minimize the peak power } \\
\text { and energy losses }\end{array}$ & $\begin{array}{l}23 \mathrm{kV} \text { Carolina } \\
\text { Power \& Light } \\
\text { Co. sys. }\end{array}$ \\
\hline 1995 & [137] & Switched & Location & Variable loads & $\begin{array}{l}\text { Dynamic Programming } \\
\text { Techniques }\end{array}$ & $\begin{array}{l}\text { Power loss minimization \& } \\
\text { network reconfiguration }\end{array}$ & $\begin{array}{l}\text { 20kV, 63-node } \\
\text { dist. Feeder }\end{array}$ \\
\hline 1996 & {$[145]$} & Switched & Location & $\begin{array}{l}\text { Different load } \\
\text { conditions }\end{array}$ & $\begin{array}{l}\text { A Neural Network } \\
(\mathrm{NN})\end{array}$ & $\begin{array}{l}\text { Minimize } \mathrm{I}^{2} \mathrm{R} \text { losses and } \\
\text { maintain all bus voltages }\end{array}$ & 30-bus \\
\hline 2002 & {$[135]$} & Fixed & Location & Average load & MNV \& GA algorithm & Power loss reduction & 69-bus \\
\hline 2006 & [146] & Fixed & $\begin{array}{l}\text { Location } \\
+ \text { Size }\end{array}$ & $\begin{array}{l}\text { Non-linear and } \\
\text { Unbalanced Loads }\end{array}$ & Genetic Algorithm & $\begin{array}{l}\text { Minimize power loss and } \\
\text { harmonic distortion }\end{array}$ & 34-bus \\
\hline 2008 & [136] & Switched & Location & Average load & $\begin{array}{l}\text { Ant Colony Search } \\
\text { Algorithm (ACSA). }\end{array}$ & Minimize power loss & $\begin{array}{l}\text { 3-feeder dist. } \\
\text { System }\end{array}$ \\
\hline 2009 & [125] & Fixed & $\begin{array}{l}\text { Location } \\
+ \text { Size }\end{array}$ & $\begin{array}{l}\text { Linear and } \\
\text { nonlinear load } \\
\text { models }\end{array}$ & $\begin{array}{l}\text { Genetic Algorithms } \\
\text { (GA) }\end{array}$ & $\begin{array}{l}\text { Power and energy Losses } \\
\text { minimization }\end{array}$ & $\begin{array}{l}11 \mathrm{kV}, 30 \text {-node } \\
\text { feeder }\end{array}$ \\
\hline 2010 & [138] & Fixed & Location & $\begin{array}{l}\text { Different load } \\
\text { levels }\end{array}$ & $\begin{array}{l}\text { Mixed-integer non- } \\
\text { linear programming }\end{array}$ & Minimize the energy loss & $16,33 \& 83$-bus \\
\hline 2011 & [134] & Fixed & Location & Average load & Harmony Search (HA) & $\begin{array}{l}\text { Minimization of losses cost } \\
\text { and reliability cost }\end{array}$ & 83-bus \\
\hline 2012 & {$[124]$} & Switched & Location & $\begin{array}{l}\text { Different load } \\
\text { levels }\end{array}$ & SAIDI, SAIFI & $\begin{array}{l}\text { Minimize capacitor } \\
\text { investment \& energy cost }\end{array}$ & $\begin{array}{l}\text { Tabriz power } \\
\text { electric dist., Iran }\end{array}$ \\
\hline 2012 & {$[147]$} & Both & Location & $\begin{array}{l}\text { Different load } \\
\text { levels }\end{array}$ & GA and OPF & Multi objectives & 70-bus \\
\hline 2013 & {$[123]$} & Fixed & $\begin{array}{l}\text { Location } \\
+ \text { Size } \\
\end{array}$ & $\begin{array}{l}\text { Different load } \\
\text { levels }\end{array}$ & Memetic algorithm & $\begin{array}{l}\text { Minimize power loss and } \\
\text { improve voltage profiles }\end{array}$ & 34-bus \\
\hline 2013 & [148] & Fixed & $\begin{array}{l}\text { Location } \\
+ \text { Size }\end{array}$ & $\begin{array}{l}\text { Different load } \\
\text { levels }\end{array}$ & Mixed-integer LP & $\begin{array}{l}\text { Minimize power loss } \\
\text { and improve voltage } \\
\text { profiles }\end{array}$ & $136 \& 69-$ bus \\
\hline
\end{tabular}


S. M. G. Mostafa et. al. /JEA Vol. 01(04) 2020, pp 150-169

\begin{tabular}{|c|c|c|c|c|c|c|c|}
\hline $\begin{array}{l}\text { Publi- } \\
\text { shed }\end{array}$ & Ref. & $\begin{array}{l}\text { SCB } \\
\text { type }\end{array}$ & $\begin{array}{l}\text { Design } \\
\text { variables }\end{array}$ & Load profiles & Method & Objective function & Test systems \\
\hline 2013 & {$[141]$} & Both & $\begin{array}{l}\text { Location } \\
+ \text { Size }\end{array}$ & $\begin{array}{l}\text { Different load } \\
\text { levels }\end{array}$ & $\begin{array}{l}\text { Modified Discrete } \\
\text { PSO }\end{array}$ & $\begin{array}{l}\text { Minimize capacitor } \\
\text { investment \& energy cost }\end{array}$ & 33, 37-bus \\
\hline 2014 & [126] & Switched & $\begin{array}{l}\text { Location } \\
+ \text { Size } \\
\end{array}$ & $\begin{array}{l}\text { Different load } \\
\text { levels }\end{array}$ & $\begin{array}{l}\text { ICA/GA hybrid } \\
\text { method }\end{array}$ & Multi objectives & $33 \& 69$-bus \\
\hline 2014 & {$[127]$} & Fixed & Size & $\begin{array}{l}\text { Linear and non- } \\
\text { linear loads }\end{array}$ & Genetic algorithm & $\begin{array}{l}\text { Minimize THD, power loss } \\
\& \text { improve voltage profiles }\end{array}$ & 33-bus \\
\hline 2015 & {$[128]$} & Fixed & $\begin{array}{l}\text { Location } \\
+ \text { Size }\end{array}$ & $\begin{array}{l}\text { Uncertain load } \\
\text { variations }\end{array}$ & MOPSO method & $\begin{array}{l}\text { Minimize power loss and } \\
\text { improve bus voltage }\end{array}$ & 33, 94-bus \\
\hline 2016 & [129] & Switched & $\begin{array}{l}\text { Location } \\
+ \text { Size }\end{array}$ & Average load & IMDE algorithm & Minimize power loss & 33,69 -bus \\
\hline 2017 & [139] & Both & $\begin{array}{l}\text { Location } \\
+ \text { Size }\end{array}$ & $\begin{array}{l}\text { Discrete load } \\
\text { levels }\end{array}$ & HS-PABC algorithm & $\begin{array}{l}\text { Minimize power loss and } \\
\text { improve bus voltage }\end{array}$ & 69,118 -bus \\
\hline 2017 & {$[130]$} & Fixed & $\begin{array}{l}\text { Location } \\
+ \text { Size }\end{array}$ & $\begin{array}{l}\text { Two Different } \\
\text { load level }\end{array}$ & IVM \& PLI algorithm & Minimize power loss & 33,85 -bus \\
\hline 2017 & [131] & Switched & $\begin{array}{l}\text { Location } \\
+ \text { Size } \\
\end{array}$ & $\begin{array}{l}\text { Variable load } \\
\text { levels }\end{array}$ & MOEA/D algorithm & $\begin{array}{l}\text { Minimizing system real } \\
\text { and reactive power losses. }\end{array}$ & $\begin{array}{l}33,69,83,119- \\
\text { bus }\end{array}$ \\
\hline 2018 & {$[150]$} & Switched & $\begin{array}{l}\text { Location } \\
+ \text { Size }\end{array}$ & Average load & $\begin{array}{l}\text { Water Cycle } \\
\text { Algorithm }\end{array}$ & $\begin{array}{l}\text { Minimizing power losses, } \\
\text { voltage deviation, } \\
\text { electrical energy cost, total } \\
\text { emissions }\end{array}$ & $\begin{array}{l}33,69 \text {-bus \& real } \\
\text { Egyptian system }\end{array}$ \\
\hline 2018 & {$[140]$} & Fixed & $\begin{array}{l}\text { Location } \\
+ \text { Size }\end{array}$ & Average load & $\begin{array}{l}\text { Multi-Objective } \\
\text { Optimization Problem }\end{array}$ & $\begin{array}{l}\text { Minimized losses \& } \\
\text { reduced voltage } \\
\text { unbalancing }\end{array}$ & $\begin{array}{l}\text { IEEE-37 and } \\
123 \text {-node }\end{array}$ \\
\hline 2019 & [151] & Fixed & $\begin{array}{l}\text { Location } \\
+ \text { Size } \\
\end{array}$ & $\begin{array}{l}\text { Two load power } \\
\text { factor }\end{array}$ & $\begin{array}{l}\text { Water Cycle } \\
\text { Algorithm (WCA) }\end{array}$ & $\begin{array}{l}\text { Minimize power loss and } \\
\text { improve bus voltage }\end{array}$ & 33-bus \\
\hline 2019 & {$[152]$} & Fixed & $\begin{array}{l}\text { Location } \\
+ \text { Size }\end{array}$ & Average load & $\begin{array}{l}\text { GA interfaced with } \\
\text { COM model }\end{array}$ & $\begin{array}{l}\text { Minimize power loss and } \\
\text { improve voltage profiles }\end{array}$ & IEEE-13,37-bus \\
\hline 2019 & [153] & Switched & $\begin{array}{l}\text { Location } \\
+ \text { Size } \\
\end{array}$ & Average load & $\begin{array}{l}\text { Hybrid WIPSO-GSA } \\
\text { algorithm }\end{array}$ & $\begin{array}{l}\text { Maximization of total cost } \\
\text { benefit }\end{array}$ & $\begin{array}{l}\text { 33-bus \& Indian } \\
\text { 85-bus }\end{array}$ \\
\hline 2019 & [154] & Both & Location & $\begin{array}{l}\text { Voltage-dependent } \\
\text { load }\end{array}$ & $\begin{array}{l}\text { The mixed-integer } \\
\text { second-order cone } \\
\text { programming model }\end{array}$ & $\begin{array}{l}\text { Minimize power loss and } \\
\text { improve voltage profiles }\end{array}$ & $\begin{array}{l}\text { 69,2313-node } \\
\text { dist. Sys. }\end{array}$ \\
\hline 2019 & {$[155]$} & Fixed & $\begin{array}{l}\text { Location } \\
+ \text { Size }\end{array}$ & $\begin{array}{l}\text { Different load } \\
\text { condition }\end{array}$ & $\begin{array}{l}\text { Sensitivity analysis } \\
\text { based on voltage } \\
\text { stability index }\end{array}$ & $\begin{array}{l}\text { Minimize feeder current, } \\
\text { power loss and improve } \\
\text { voltage profiles }\end{array}$ & $\begin{array}{l}\text { 33-bus dist. } \\
\text { System }\end{array}$ \\
\hline
\end{tabular}

\subsection{Evaluation of the Methods}

It is easy for the implementation of the analytical method, and its execution is faster. Since it takes a simple presumption and considers one snapshot of an electric power system loading condition, their results are suggestive. The strength of the Exhaustive Search (ES) method is -it is assured to the finding of global optimum, but it does not itself a simulation technique and appropriate for the large electric system.

Hence, in a dynamic programming method, this ES method is not suitable. All Heuristic methods are robust. It can furnish very accurate solutions for optimal SCBs placement for large and complex systems. They needed huge computations.

Nevertheless, this drawback is not essential that much critical in the applications of SCBs placement. The most frequently applied methods are AI methods for SCBs placement because it finds optimum solutions very fast. Most of the current researches is running based on AI methods and employed in Multi-dimensional problem solutions for their accuracy and fast convergence characteristics.

\section{Combinatorial Method}

The Combinatorial Method (CM) is for radial distribution system with source (substation) bus as slack bus and all other load buses taken as PQ buses. The algorithm proposed is described in the following steps shown in Fig. 3 for deciding the optimal sizes of the capacitors in terms of standard sizes available in the market and their locations (only load buses):

(i). Input Data and Initialization: The distribution system data is initialized in this step

(ii). Base Case Results: The "Forward/Backward Sweep" method of the Deterministic Load Flow (DLF) is carried out for the base case study to store the base case results, which will be used to compare the results with (1).

(iii). Generation of Combinations: All possible combinations of different commercially available capacitors are generated. Similarly, all possible combinations of the node are created.

(iv). Capacitor Placement: Each capacitor of the first combination is kept at corresponding load buses of the first combination of node and run the DLF to get the feeder loss. Similarly, DLF is performed to get the loss by placing capacitors 
from the first combination to the second, third until the last combination of nodes, and getting the losses. After finishing, the second combination of the capacitor is placed to all combinations corresponding to the same previous procedures to get the losses. This procedure is repeated for all capacitors combination.

(v). The program is terminated when DLF is performed at all node combinations by each capacitor of all capacitor combinations. Finally, the minimum feeder loss and a corresponding combination of the capacitor and node are determined.

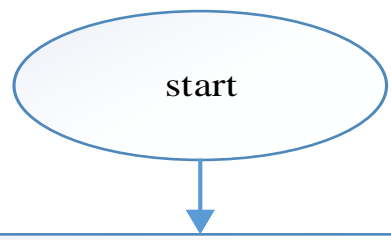

Read Distribution System Data (22, 69 bus data)

Read discrete size of capacitors Standard capacitors size is multiple of $150 \mathrm{kVAr}$

Generate random combinations of node and capacitors

Run DLF by connecting each
combination of capacitors to each
combination of node to get the
minimum feeder loss

Print the minimum loss and regarding combination of capacitors and node

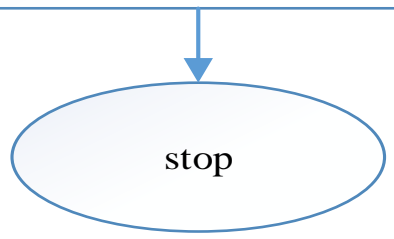

Fig. 3 Algorithm of the combinatorial method based capacitor placement.

Since every capacitor combination is checked with all node combinations, the program needs huge computational time. Still, it has given more accurate results comparatively with another capacitor placement algorithm. In this study, two standard test systems are considered for analysis and demonstrating the above algorithm with practical Indian 22-bus and IEEE 69-bus system.

Standard capacitor sizes available in the literature (in $\mathrm{kVAr}$ ): 150, 300, 450, 600, 750, 900, 1050, 1200, 1350, 1500, 1650, $1800,1950,2100,2250,2400,2550,2700,2850,3000,3150$, $3300,3450,3600,3750,3900,4050$.

\subsection{2-bus Radial Practical Test System}

The data for the 22-bus agricultural test system is given in [67]. This 22-bus system belongs to a small part of India's Eastern Power Distribution system with $11 \mathrm{kV}$ base voltage. It has a $662.311 \mathrm{~kW}$ real power load and $667.40 \mathrm{kVAr}$ reactive power load comprised of 21 branches and 22-buses in Fig. 4. This practical test system is rated with voltage $11 \mathrm{kV}, \mathrm{Vmax}=1.1$ $\mathrm{pu}$, and $\mathrm{Vmin}=0.9 \mathrm{pu}$, along with a base $10 \mathrm{MVA}$ complex power rating.

The optimal locations are found at node-9, 13, 17, and node20 with $150 \mathrm{kVAr}$ in every node for the nominal load (100\%) after completing the simulation using the proposed combinatorial method. The minimum loss is $9.30 \mathrm{~kW}$, and the lowest voltage is $0.9817 \mathrm{pu}$ at node-22, but for the light load (50\%) condition, the loss became $2.39 \mathrm{~kW}$ that have $0.9904 \mathrm{pu}$ voltage at node-22 while optimal location found node- 9 and node- 17 with $150 \mathrm{kVAr}$ each. Besides, for peak load (160\%) condition, feeder loss is $24.41 \mathrm{~kW}$, and lowest voltage at node is 22 with 0.9700 pu using a total of $900 \mathrm{kVAr}$ capacitor bank in four optimal locations (Table 7).

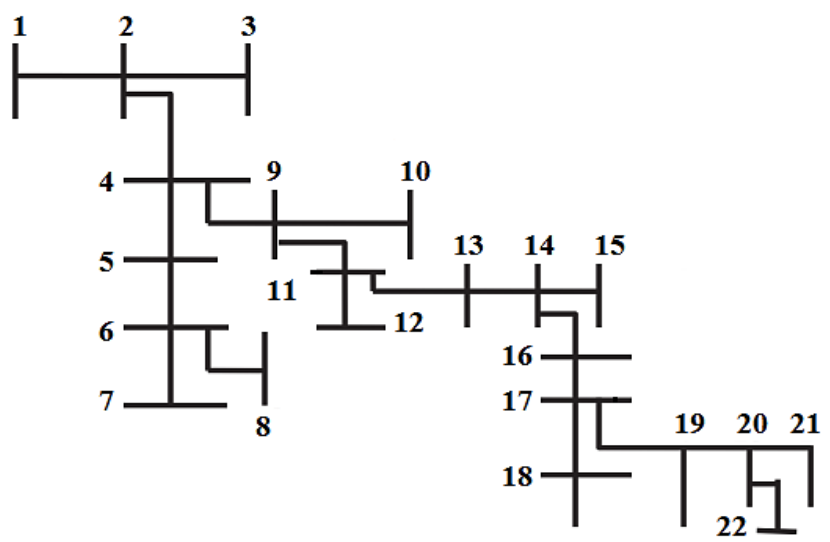

Fig. 4 22-bus agricultural practical Indian agricultural test system.

The simulated results are compared with DSA [67], [95]. The optimal locations and sizes have appended in Table 7, including feeder loss and lowest voltage level considering three different loading conditions such as nominal load (100\%), light load $(50 \%)$, and peak load (160\%). One year or 8760 hours have been divided into 5260 hours for nominal load, 2000 hours for a light load, and 1500 hours for peak load.

Table 6 Load level and load duration time.

\begin{tabular}{|l|c|c|c|}
\hline Load level & 0.5 (light) & $\begin{array}{l}1.0 \\
\text { (normal) }\end{array}$ & 1.6 (peak) \\
\hline Duration (hr) & 2000 & 5260 & 1500 \\
\hline
\end{tabular}

The real power loss in the whole feeder is $17.7 \mathrm{~kW}, 4.30$ $\mathrm{kW}$, and $46.08 \mathrm{~kW}$ for nominal, light, and peak load, respectively, by the analytical method without using any capacitor compensation. It is found that simulations carried out using Combinatorial Method provide a better total cost, cost of energy loss, and cost of capacitor installation than that obtained from the Direct Search Algorithm (DSA) found in the literature. Also, it is seen that there is more minimization in power loss in nominal (100\%) and peak (160\%) load conditions with respect to DSA and TLBO, but voltage level reduced a little bit in every load condition. 
Table 7 Real power loss and voltage profile with different load scenarios in 22-bus.

\begin{tabular}{|c|c|c|c|c|c|c|}
\hline \multirow{2}{*}{$\begin{array}{l}\text { 22-bus system } \\
\text { 1. Nominal load (100\%) } \\
\text { Optimal Placement }\end{array}$} & \multicolumn{2}{|l|}{ DSA [67] } & \multicolumn{2}{|c|}{ TLBO [95] } & \multicolumn{2}{|c|}{ Combinatorial Method (CM) } \\
\hline & Location & $\begin{array}{l}\text { Size } \\
(\mathrm{kVAr})\end{array}$ & Location & $\begin{array}{l}\text { Size } \\
\text { (kVAr) }\end{array}$ & Location & Size (kVAr) \\
\hline & 4 & 150 & 9 & 150 & 9 & 150 \\
\hline & 13 & 300 & 14 & 150 & 13 & 150 \\
\hline & 16 & 150 & 17 & 150 & 17 & 150 \\
\hline & 17 & 150 & 20 & 150 & 20 & 150 \\
\hline Minimum voltage node & \multicolumn{2}{|l|}{22} & \multicolumn{2}{|l|}{22} & \multicolumn{2}{|l|}{22} \\
\hline Minimum voltage (pu) & \multicolumn{2}{|l|}{0.9824} & \multicolumn{2}{|l|}{0.9822} & \multicolumn{2}{|l|}{0.9817} \\
\hline Power Loss $(\mathrm{kW})$ & \multicolumn{2}{|l|}{9.66} & \multicolumn{2}{|l|}{9.31} & \multicolumn{2}{|l|}{9.30} \\
\hline \multirow{4}{*}{$\begin{array}{l}\text { 2. Light load (50\%) } \\
\text { Optimal Placement }\end{array}$} & 4 & 0 & 9 & 150 & 9 & 150 \\
\hline & 13 & 150 & 14 & 0 & 13 & 0 \\
\hline & 16 & 150 & 17 & 150 & 17 & 150 \\
\hline & 17 & 0 & 20 & 0 & 20 & 0 \\
\hline Minimum voltage node & \multicolumn{2}{|l|}{22} & \multicolumn{2}{|l|}{22} & \multicolumn{2}{|l|}{22} \\
\hline Minimum voltage $(\mathrm{pu})$ & \multicolumn{2}{|l|}{0.9909} & \multicolumn{2}{|l|}{0.9903} & \multicolumn{2}{|l|}{0.9904} \\
\hline Power Loss $(\mathrm{kW})$ & \multicolumn{2}{|l|}{2.39} & 2.39 & & \multicolumn{2}{|l|}{2.39} \\
\hline \multirow{4}{*}{$\begin{array}{l}\text { 3. Peak load (160\%) } \\
\text { Optimal Placement }\end{array}$} & 4 & 150 & 9 & 150 & 9 & 150 \\
\hline & 13 & 450 & 14 & 300 & 13 & 300 \\
\hline & 16 & 300 & 17 & 150 & 17 & 150 \\
\hline & 17 & 150 & 20 & 300 & 20 & 300 \\
\hline Minimum voltage node & \multicolumn{2}{|l|}{22} & \multicolumn{2}{|l|}{22} & \multicolumn{2}{|l|}{22} \\
\hline Minimum voltage $(\mathrm{pu})$ & \multicolumn{2}{|l|}{0.9701} & \multicolumn{2}{|l|}{0.9712} & \multicolumn{2}{|l|}{0.9700} \\
\hline Power Loss $(\mathrm{kW})$ & \multicolumn{2}{|l|}{24.89} & \multicolumn{2}{|l|}{24.43} & \multicolumn{2}{|l|}{24.41} \\
\hline $\begin{array}{l}\text { Ratings of the installed } \\
\text { capacitor (maximum one) } \\
\text { kVAr) }\end{array}$ & $\begin{array}{l}150,450, \\
\text { (Total }=10\end{array}$ & 0,150 & $\begin{array}{l}150,300 \\
\text { (Total }=9\end{array}$ & & 150,300 & $300($ Total $=900)$ \\
\hline Capacitor cost $(\$)$ & $1050 * 3=3$ & & $900 * 3=2$ & & $900 * 3=2$, & \\
\hline The energy lost cost (\$) & 5575.59 & & 5421.53 & & 5421.53 & \\
\hline Total cost with capacitor $(\$)$ & $8,725.59$ & & $8,121.53$ & & $8,121.53$ & \\
\hline
\end{tabular}

Comparison of voltage profiles has demonstrated in Fig. 5 for different loading conditions. It is seen that the voltage level has improved due to the employment of SCBs except for peak load conditions. It is because extra loads cause more voltage deviation than nominal load, and without reactive compensation, the scenario will be worse. Comparison of percentage improvement of voltage profile and total feeder loss minimization for DSA, TLBO, and CM methods have shown in Fig. 6. There are remarkable improvements in power loss reduction after SCBs connection where CM gives $47.42 \%$ loss reduction than without $\mathrm{SCB}$ compensation. This figure is much better than DSA (45.39\%) and TLBO (47.37\%) at nominal load. A similar loss reduction pattern has been maintained for light load and peak load conditions. Voltage profiles also improved, but in peak load, the condition the amount is appreciable than nominal and load. CM shows more voltage level enhancement than DSA and TLBO in the 22-bus distribution system.

Per unit cost of energy has taken $\$ 0.06 / \mathrm{kWh}$, and the cost of capacitor bank has been considered \$3.0/ $\mathrm{kVAr}$ [67] in cost calculations. Without installing the capacitor bank, the total cost of energy loss in different load conditions is $\$ 10,249.32 /$ year. After using capacitors, it became \$8,121.53/year that saved $\$ 2,127.79$ annually, and this amount is better than the DSA $(\$ 8,725.59 /$ year) techniques (Table 7$)$.

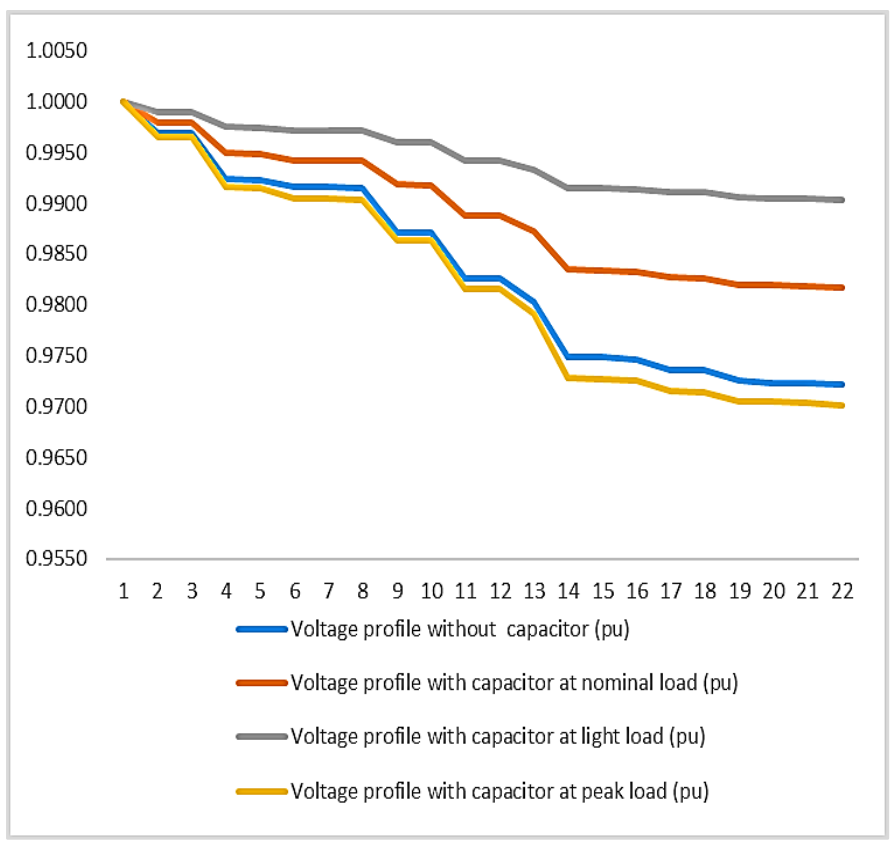

Fig. 5 The contrast of voltage profile employing SCBs at different loading conditions. 


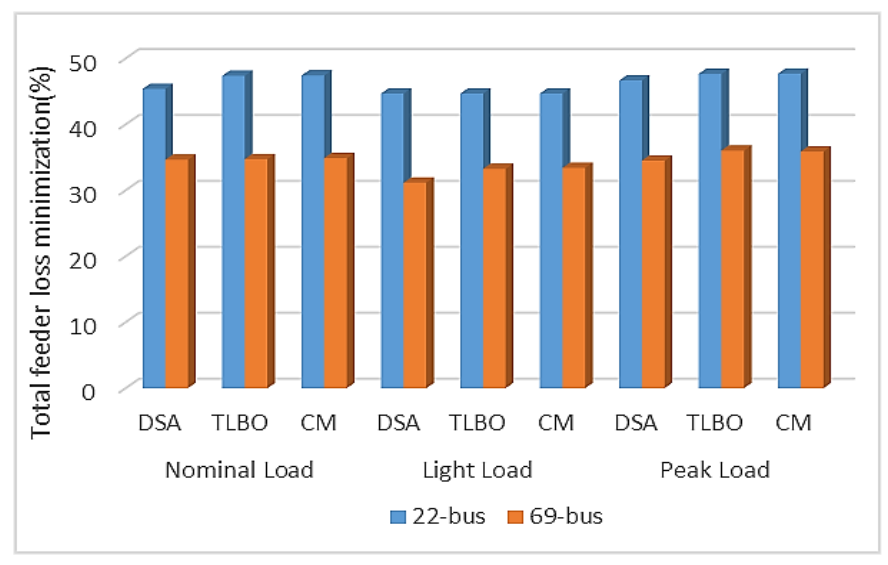

(a)

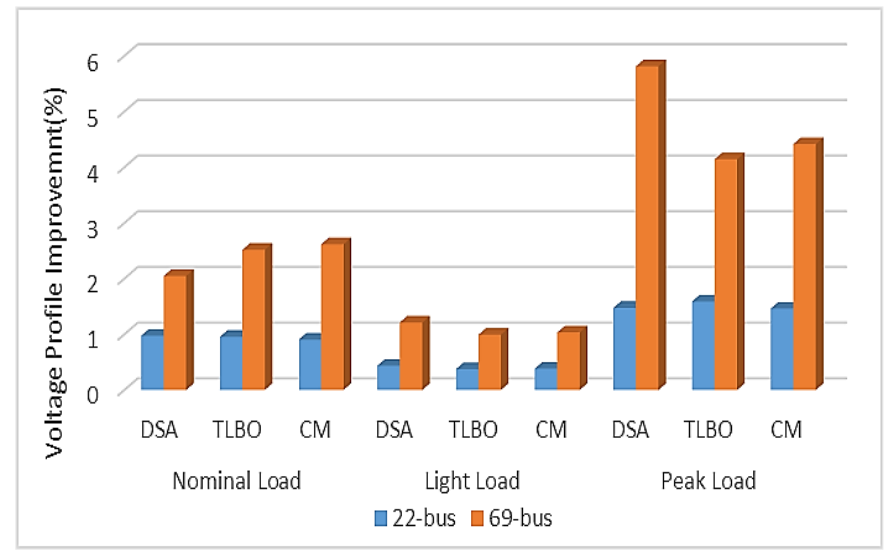

(b)

Fig. 6 The contrast of 3-different methods of SCBs sitting: (a) Percentage of line loss reduction, (b) Percentage of VPI.

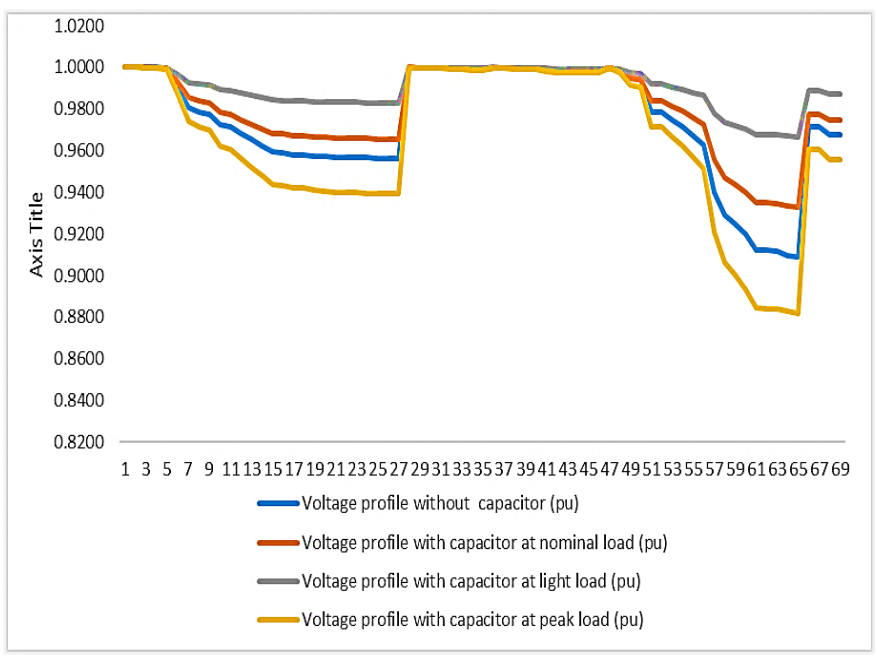

Fig. 7 The contrast of voltage profile employing SCBs at different loading conditions.

\subsection{9-bus Radial Practical Test Systems}

The complete system data has been taken from D. Das et al., 2008. This 69-bus radial test system comprises 69 nodes and 68 branches with base MVA 10 MVAr and $12.7 \mathrm{kV}$ base voltage and a total of $1.896 \mathrm{MW}$ real power load and 1.347 MVAr reactive power loads. The maximum voltage rating is $\mathrm{Vmax}=1.1$ $\mathrm{pu}$, and the minimum voltage rating is $\mathrm{Vmin}=0.9 \mathrm{pu}$. This radial test system's real power loss is $225 \mathrm{~kW}$ [87] without employing any reactive power compensation. Simulation using the backward-forward sweep method obtained the same results.

Different groupings of $150 \mathrm{kVAr}, 300 \mathrm{kVAr}, 450 \mathrm{kVAr}, 750$ $\mathrm{kVAr}$, and $1050 \mathrm{kVAr}$ commercially available static capacitors have been used to generate combinations using three optimal places to get the lowest real power loss and voltage profile. Node-61, 64, 18 with $1050 \mathrm{kVAr}, 300 \mathrm{kVAr}$, and $300 \mathrm{kVAr}$ rating gives the minimum $146.50 \mathrm{~kW}$ loss that maintains 0.9330 pu voltage level that is a better result than Fuzzy GA [87] and DSA [67].

The simulated output using the combinatorial method and backward-forward power flow is assessed with DSA and Fuzzy GA. The minimum loss locations and sizes are given in Table 8, considering three different loadings. The real power loss $146.50 \mathrm{~kW}$ for the nominal load (100\%), $34.36 \mathrm{~kW}$ for the light load (50\%), and $417.60 \mathrm{~kW}$ for peak load (160\%) with no additional reactive power supply. It is observed that simulation performing with combinatorial method furnished relatively than Fuzzy GA and DSA. Besides, it is found that there is more minimization in power loss in nominal and light load conditions rather than GA and DSA techniques. Meanwhile, the voltage profile is slightly decreased in light and peak load but shows an improved level than in nominal load condition ( 0.9330 p.u.) than DSA. Without installing the capacitor bank, the total cost of feeder energy loss in various loading conditions is $\$ 135,936.00 /$ year (Table 8). After using capacitors, it became $\$ 87,999.3 /$ year that saved $\$ 41,636.70$ annually, and this amount is better than the Fuzzy GA and DSA techniques.

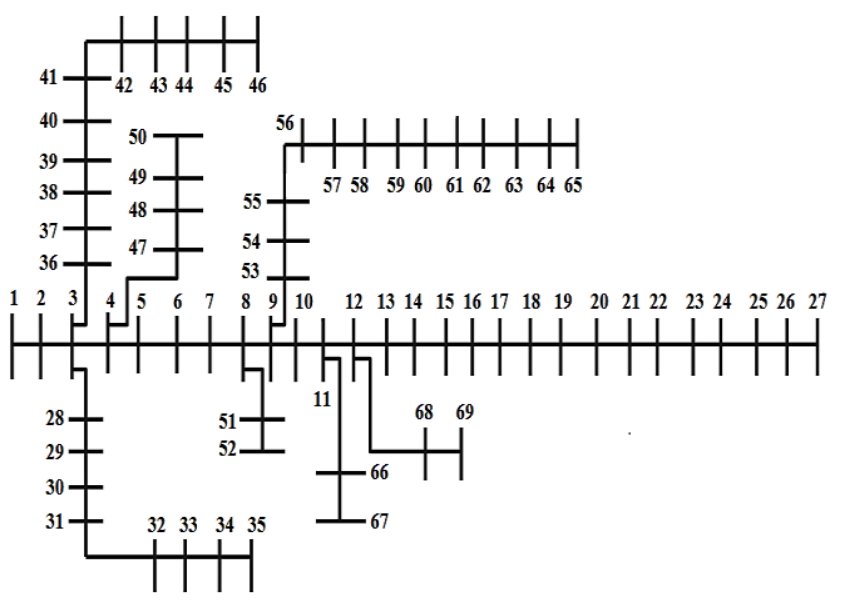

Fig. 8 69-bus radial distribution test system.

Like a 22-bus distribution system, voltage improves significantly in the 69-bus test system after employing SCBs except for peak load, where nominal load provides better VPI (Fig. 7). Moreover, Fig. 6 (a) shows the percentage of feeder loss minimization for 69 -bus distribution feeder. CM provides a better loss of minimization than DSA and TLBO. For nominal load and light, load CM provides $34.89 \%$ and $33.41 \%$ loss reduction, where DSA and TLBO provide $34.65 \%, 34.74 \%$, and $31.16 \%$, $33.27 \%$, respectively. But for peak load condition, TLBO shows better loss reduction (36.04\%) than CM (35.89\%). The percentage VPI for the 69-bus system is much better than the 22-bus radial system. It is seen from Fig. 6 that for nominal load level CM gives better bus voltage improvement compare to DSA and TLBO were $2.62 \%, 2.52 \%, 2.05 \%$ VPI for CM, DSA, and TLBO. 
Table 8 Real power loss and voltage profile with different load scenarios in 69-bus.

\begin{tabular}{|c|c|c|c|c|c|c|c|c|}
\hline 69-bus system & \multicolumn{2}{|c|}{ Fuzzy GA [87] } & \multicolumn{2}{|l|}{ DSA [67] } & \multicolumn{2}{|c|}{ TLBO [95] } & \multicolumn{2}{|c|}{$\begin{array}{l}\text { Combinatorial } \\
\text { Method (CM) }\end{array}$} \\
\hline \multirow{4}{*}{$\begin{array}{l}\text { 1. Nominal load } \\
(100 \%) \\
\text { Optimal Placement }\end{array}$} & Location & $\begin{array}{l}\text { Size } \\
(k V A r)\end{array}$ & Location & $\begin{array}{l}\text { Size } \\
(\mathrm{kVAr})\end{array}$ & Location & $\begin{array}{l}\begin{array}{l}\text { Size } \\
(\mathrm{kVAr})\end{array} \\
\end{array}$ & Location & $\begin{array}{l}\text { Size } \\
(\mathrm{kVAr})\end{array}$ \\
\hline & 59 & 100 & 15 & 450 & 22 & 300 & 61 & 1050 \\
\hline & 61 & 700 & 60 & 450 & 61 & 1050 & 64 & 300 \\
\hline & 64 & 800 & 61 & 900 & 62 & 300 & 18 & 300 \\
\hline $\begin{array}{l}\text { Minimum voltage } \\
\text { node }\end{array}$ & \multicolumn{2}{|l|}{65} & \multicolumn{2}{|l|}{65} & \multicolumn{2}{|l|}{65} & \multicolumn{2}{|l|}{65} \\
\hline Minimum voltage $(\mathrm{pu})$ & \multicolumn{2}{|l|}{0.93693} & \multicolumn{2}{|l|}{0.9318} & \multicolumn{2}{|l|}{0.9321} & \multicolumn{2}{|l|}{0.9330} \\
\hline Power Loss $(\mathrm{kW})$ & \multicolumn{2}{|l|}{156.52} & \multicolumn{2}{|l|}{147.00} & \multicolumn{2}{|l|}{146.80} & \multicolumn{2}{|l|}{146.50} \\
\hline \multirow{3}{*}{$\begin{array}{l}\text { 2. Light load }(50 \%) \\
\text { Optimal Placement }\end{array}$} & 59 & 0.00 & 15 & 300 & 22 & 150 & 61 & 450 \\
\hline & 61 & 0.00 & 60 & 300 & 61 & 450 & 64 & 150 \\
\hline & 64 & 300 & 61 & 450 & 62 & 150 & 18 & 150 \\
\hline $\begin{array}{l}\text { Minimum voltage } \\
\text { node }\end{array}$ & \multicolumn{2}{|l|}{65} & \multicolumn{2}{|l|}{65} & \multicolumn{2}{|l|}{65} & \multicolumn{2}{|l|}{65} \\
\hline Minimum voltage $(\mathrm{pu})$ & \multicolumn{2}{|l|}{0.9622} & \multicolumn{2}{|l|}{0.9683} & \multicolumn{2}{|l|}{0.9662} & \multicolumn{2}{|l|}{0.9666} \\
\hline Power Loss $(\mathrm{kW})$ & \multicolumn{2}{|l|}{40.48} & \multicolumn{2}{|l|}{35.52} & \multicolumn{2}{|l|}{34.43} & \multicolumn{2}{|l|}{34.36} \\
\hline \multirow{3}{*}{$\begin{array}{l}\text { 3. Peak load (160\%) } \\
\text { Optimal Placement }\end{array}$} & 59 & 1100 & 15 & 900 & 22 & 300 & 61 & 1050 \\
\hline & 61 & 800 & 60 & 900 & 61 & 1050 & 64 & 750 \\
\hline & 64 & 1200 & 61 & 1800 & 62 & 750 & 18 & 300 \\
\hline $\begin{array}{l}\text { Minimum voltage } \\
\text { node }\end{array}$ & \multicolumn{2}{|l|}{65} & \multicolumn{2}{|l|}{65} & 65 & & 65 & \\
\hline Minimum voltage $(\mathrm{pu})$ & 0.90014 & & 0.8936 & & 0.8795 & & 0.8818 & \\
\hline Power Loss $(\mathrm{kW})$ & 460.45 & & 427.30 & & 417.28 & & 417.60 & \\
\hline $\begin{array}{l}\text { Ratings of the } \\
\text { installed capacitor } \\
\text { (maximum one) } \\
\text { kVAr) }\end{array}$ & $\begin{array}{l}1100,800 \\
\text { (Total }=31\end{array}$ & $\begin{array}{l}\text { 1200, } \\
00)\end{array}$ & $\begin{array}{l}\text { 900, 900, } \\
\text { (Total }=36\end{array}$ & $\begin{array}{l}\text { 1800 } \\
00)\end{array}$ & $\begin{array}{l}1050,750 \\
\text { (Total }=21\end{array}$ & & $\begin{array}{l}1050,750, \\
\text { (Total }=21\end{array}$ & \\
\hline Capacitor cost (\$) & $3100 * 3=c$ & 300 & $3600 * 3=1$ & 0,800 & $3150 * 3=6$ & & $3150 * 3=6$ & \\
\hline $\begin{array}{l}\text { The energy lost cost } \\
\text { (\$) }\end{array}$ & 95727.00 & & $89,112.60$ & & $87,999.30$ & & $87,999.30$ & \\
\hline $\begin{array}{l}\text { Total cost with } \\
\text { capacitor }(\$)\end{array}$ & $105,027.0$ & & $99,912.60$ & & $94,299.30$ & & $94,299.30$ & \\
\hline
\end{tabular}

\section{Conclusion}

In this paper, the second section has presented an in-depth comparative review of SCBs placement and sizing that included types, design variables, load profiles, methods, and test distribution systems sequentially through classification and analyzation of present and future trends. There are four types of SCBs problems that have been reviewed; however, Analytical methods and Numerical methods have provided the most robust solution, but these methods needed higher computational time. Contrary, AI methods seek the optimum solution that depends on the searching ability of the algorithm hence save computational time. The most frequently applied methods are the AI method for SCBs placement in recent research due to its computational characteristics.

A new approach called Combinatorial Method has developed for optimal placement and sizing of SCBs in distribution systems in the third section. The locations and sizes have been determined by generating random combinations and running deterministic load flow each time. The results obtained from the proposed technique have been compared with DSA and
FGA, and TLBO algorithm. The research study has been carried out on modified Indian practical 22-bus and IEEE 69-bus system. The results showed that around forty-seven percent loss minimized in the 22-bus system, and almost thirty-five percent loss was reduced in 69-bus radial distribution systems. Besides, reactive compensation still maintains a satisfactory voltage level at all buses and SCB connection points. The proposed algorithm saved more in terms of money annually than the DSA and Fuzzy GA and TLBO method by optimal sizing and sitting of SCBs. Though the proposed $\mathrm{CM}$ method is time-consuming, this method would help the researcher achieve better results for planning purposes. Due to feeder loss minimization and voltage profile improvement in distribution feeders, both utilities and individual owners will be encouraged to accommodate more DGs.

\subsection{Future Study}

Though many works are already done for optimal sitting and sizing of SCBs, further research is necessary to enhance the performance and capability of SCBs to solve more complex 
problems introduced by renewable energy integration on the existing grid. Wind velocity and solar radiations are not the only uncertain parameters, but there are other Distributed Energy Resources (DERs) and metrics that are stochastic such as: market price, future capital cost, fuel price, future fuel supply system, future load growth, and power of plug-in Electric Vehicles (EVs). Moreover, Network reconfiguration, optimal sitting, and sizing of DGs, Protective device placement, optimal allocation of Energy Storage System (ESS), substation, and line expansion also need to investigate simultaneously with optimal SCBs sitting and sizing. However, in the optimal SCBs placement problem, ancillary services should be considered. Because to maintain reliable grid operation, optimal SCBs placement can provide ancillary services by supplying necessary reactive power to the grid when needed. Finally, more robust and fast programming methods are required that give more accurate measures with minimum memory requirement.

\section{Acknowledgments}

The first author would like to thanks ADB-JSP for providing the scholarship to pursue his master's degree and carry out this work at the Asian Institute of Technology, Thailand.

\section{References}

[1] Arulraj, R. and Kumarappan, N., 2019. Optimal economic-driven planning of multiple DG and capacitor in distribution network considering different compensation coefficients in feeder's failure rate evaluation. Engineering Science and Technology, an International Journal, 22(1), pp.67-77.

[2] Neagle, N.M. and Samson, D.R., 1956. Loss reduction from capacitors installed on primary feeders [includes discussion]. Transactions of the American Institute of Electrical Engineers. Part III: Power Apparatus and Systems, 75(3), pp.950-959.

[3] Kasztenny, B., Schaefer, J. and Clark, E., 2007, March. Fundamentals of adaptive protection of large capacitor banks. In 2007 Power Systems Conference: Advanced Metering, Protection, Control, Communication, and Distributed Resources (pp. 154-186). IEEE.

[4] Segura, S., Romero, R. and Rider, M.J., 2010. Efficient heuristic algorithm used for optimal capacitor placement in distribution systems. International journal of electrical power \& energy systems, 32(1), pp.71-78.

[5] Lidula, N.W.A. and Rajapakse, A.D., 2011. Microgrids research: A review of experimental microgrids and test systems. Renewable and Sustainable Energy Reviews, 15(1), pp.186-202.

[6] Eltawil, M.A. and Zhao, Z., 2010. Grid-connected photovoltaic power systems: Technical and potential problems-A review. Renewable and sustainable energy reviews, 14(1), pp.112-129.

[7] Taylor, C.W., 2003. Shunt Compensation for Voltage Stability. IFAC Proceedings Volumes, 36(20), pp.4348.

[8] Andersson, G., Donalek, P., Farmer, R., Hatziargyriou, N., Kamwa, I., Kundur, P., Martins, N., Paserba, J., Pourbeik, P., Sanchez-Gasca, J. and Schulz, R., 2005. Causes of the 2003 major grid blackouts in North America and Europe, and recommended means to improve system dynamic performance. IEEE transactions on Power Systems, 20(4), pp.1922-1928.

[9] Pereira, L., 2004. Cascade to black [system blackouts]. IEEE Power and Energy Magazine, 2(3), pp.54-57.

[10] Bruns, D.P., Newcomb, G.R., Miske, S.A., Taylor, C.W., Lee, G.E. and Edris, A., 2001. Shunt capacitor bank series group shorting (CAPS) design and application. IEEE Transactions on Power Delivery, 16(1), pp.24-32.

[11] Dortolina, C.A. and Nadira, R., 2005. The loss that is unknown is no loss at all: A top-down/bottom-up approach for estimating distribution losses. IEEE Transactions on Power Systems, 20(2), pp.1119-1125.

[12] Targosz, R., Belmans, R., Declercq, J., De Keulenaer, H., Furuya, K., Karmarkar, M., Martinez, M., McDermott, M. and Pinkiewicz, I., 2005. The potential for global energy savings from high efficiency distribution transformers. Leonardo Energy Transformer-European Copper Institute.

[13] EPRI. Assessment of transmission and distribution losses in New York.

[14] EIA, 2011. Annual Energy Outlook 2011. Available from 〈http://www.eia.gov/forecasts/archive/aeo11/> Last Accessed on: 20-Dec-20202. Information Administration, Washington, DC. 2011.

[15] Prada, R.B. and Souza, L.J., 1998. Voltage stability and thermal limit: constraints on the maximum loading of electrical energy distribution feeders. IEE ProceedingsGeneration, Transmission and Distribution, 145(5), pp.573-577.

[16] ENERGI. Guidelines to install, operate and maintain ht capacitors \& its associated equipment 〈http://www.energegroup.com/CapacitorManual.pdf〉.

[17] Aman, M.M., Jasmon, G.B., Mokhlis, H. and Bakar, A.H.A., 2013. Analysis of the performance of domestic lighting lamps. Energy policy, 52, pp.482-500.

[18] Milligan M, Ela E, Hein J, Schneider T, Brinkman G, Denholm P., 2012. Exploration of high-penetration renewable electricity futures. Vol. 4 of renewable electricity futures study. NREL/TP-6A20-52409-4. Golden, CO: National Renewable Energy Laboratory.

[19] EURELECTRIC. Power outages in 2003-global regulatory Network.

[20] Mostafa, S.M.G., Singh, J.G., Masrur, H. and Ullah, M.S., 2016, October. A prospective model of Bangladesh electricity market. In 2016 International Conference on Innovations in Science, Engineering and Technology (ICISET) (pp. 1-5). IEEE.

[21] Root, C.E., 2006. The future beckons [electric power industry]. IEEE Power and Energy Magazine, 4(1), pp.24-31.

[22] Singh, H., Hao, S. and Papalexopoulos, A., 1998. Transmission congestion management in competitive electricity markets. IEEE Transactions on power systems, 13(2), pp.672-680.

[23] Hemmati, R., Hooshmand, R.A. and Khodabakhshian, A., 2013. State-of-the-art of transmission expansion planning: Comprehensive review. Renewable and Sustainable Energy Reviews, 23, pp.312-319. 
[24] Baldick, R., 2004. Reactive issues- reactive power in restructured markets. IEEE Power \& Energy Magazine, 2(6), pp.14-17.

[25] Hogan, W.W., 1996. Markets in real electric networks require reactive prices. In Electricity transmission pricing and technology (pp. 143-182). Springer, Dordrecht.

[26] Aman, M.M., Jasmon, G.B., Bakar, A.H.A. and Mokhlis, H., 2012. Optimum capacitor placement and sizing for distribution system based on an improved voltage stability index. International Review of Electrical Engineering, 7(3), pp.4622-4630.

[27] Gonzalez-Longatt, F. and Fortoul, C., 2005, March. Review of the distributed generation concept: Attempt of unification. In International Conference on Renewable Energies and Power Quality (ICREPQ 05), España (pp. 16-18).

[28] Ackermann, T., Andersson, G. and Söder, L., 2001. Distributed generation: a definition. Electric power systems research, 57(3), pp.195-204.

[29] Mahmud, M.A., Hossain, M.J., Pota, H.R. and Nasiruzzaman, A.B.M., 2011, November. Voltage control of distribution networks with distributed generation using reactive power compensation. In IECON 2011-37th Annual Conference of the IEEE Industrial Electronics Society, pp. 985-990.

[30] Liew, S.N. and Strbac, G., 2002. Maximising penetration of wind generation in existing distribution networks. IEE Proceedings-Generation, Transmission and Distribution, 149(3), pp.256-262.

[31] Turitsyn, K., Sulc, P., Backhaus, S. and Chertkov, M., 2011. Options for control of reactive power by distributed photovoltaic generators. Proceedings of the IEEE, 99(6), pp.1063-1073.

[32] Rao, R.S., Narasimham, S.V.L. and Ramalingaraju, M., 2011. Optimal capacitor placement in a radial distribution system using plant growth simulation algorithm. International journal of electrical power \& energy systems, 33(5), pp.1133-1139.

[33] Mekhamer, S.F., Soliman, S.A., Moustafa, M.A. and ElHawary, M.E., 2002. Load flow solution of radial distribution feeders: a new contribution. International journal of electrical power \& energy systems, 24(9), pp.701-707.

[34] Cook, R.F., 1959. Analysis of capacitor application as affected by load cycle. Transactions of the American Institute of Electrical Engineers. Part III: Power Apparatus and Systems, 78(3), pp.950-956.

[35] Cook, R.F., 1961. Optimizing the application of shunt capacitors for reactive-volt-ampere control and loss reduction. Transactions of the American Institute of Electrical Engineers. Part III: Power Apparatus and Systems, 80(3), pp.430-441.

[36] Bae, Y.G., 1978. Analytical method of capacitor allocation on distribution primary feeders. IEEE Transactions on Power Apparatus and Systems, (4), pp.1232-1238.

[37] Grainger, J.J. and Lee, S.H., 1981. Optimum size and location of shunt capacitors for reduction of losses on distribution feeders. IEEE Transactions on Power Apparatus and Systems, (3), pp.1105-1118.
[38] Schmill, J.V., 1965. Optimum size and location of shunt capacitors on distribution feeders. IEEE Transactions on Power Apparatus and Systems, 84(9), pp.825-832.

[39] Chang, N.E., 1969. Locating shunt capacitors on primary feeder for voltage control and loss reduction. IEEE Transactions on Power Apparatus and Systems, (10), pp.1574-1577.

[40] Chang, N.E., 1972. Generalized equations on loss reduction with shunt capacitor. IEEE Transactions on Power Apparatus and Systems, (5), pp.2189-2195.

[41] Lee, S.H. and Grainger, J.J., 1981. Optimum placement of fixed and switched capacitors on primary distribution feeders. IEEE Transactions on Power Apparatus and Systems, (1), pp.345-352.

[42] Salama, M.M.A., Chikhani, A.Y. and Hackam, R., 1985. Control of reactive power in distribution systems with an end-load and fixed load condition. IEEE transactions on power apparatus and systems, (10), pp. 2779-2788.

[43] Salama, M.M.A., Mansour, E.A.A., Chikhani, A.Y. and Hackam, R., 1985. Control of reactive power in distribution systems with an end-load and varying load condition. IEEE transactions on power apparatus and systems, (4), pp.941-947.

[44] Haque, M.H., 1999. Capacitor placement in radial distribution systems for loss reduction. IEE Proceedings-Generation, Transmission and Distribution, 146(5), pp.501-505.

[45] Cho, M.Y. and Chen, Y.W., 1997. Fixed/switched type shunt capacitor planning of distribution systems by considering customer load patterns and simplified feeder model. IEE Proceedings-Generation, Transmission and Distribution, 144(6), pp.533-540.

[46] Arief, A. and Nappu, M.B., 2016. An analytical method for optimal capacitors placement from the inversed reduced jacobian matrix. Energy Procedia, 100(100), pp.307-310.

[47] Kamel, S., Hamdy, W., Abd-elgwad, S., Selim, A. and Jurado, F., 2019, March. Development of Probabilistic Power Flow Algorithm for Radial Distribution Systems with Capacitors Using Analytical Approach. In IEEE 10th International Renewable Energy Congress (IREC 2019), Sousse-Tunisia.

[48] Ihsan, M., Shahzad, M. and Ullah, N., 2019, January. Analytical Method for Optimal Reactive Power Support in Power Network. In 2019 2nd International Conference on Computing, Mathematics and Engineering Technologies (iCoMET) (pp. 1-6). IEEE.

[49] Crow, M.L., 2003. Computational methods for electric power systems. Crc Press.

[50] Saadat, H., 1999. Power system analysis (Vol. 2). McGraw-Hill.

[51] Dura, H., 1968. Optimum number, location, and size of shunt capacitors in radial distribution feeders a dynamic programming approach. IEEE Transactions on Power Apparatus and Systems, (9), pp.1769-1774.

[52] Fawzi, T.H., El-Sobki, S.M. and Abdel-halim, M.A., 1983. New approach for the application of shunt capacitors to the primary distribution feeders. IEEE Transactions on Power Apparatus and Systems, (1), pp.10-13. 
[53] Ponnavsikko, M. and Rao, K.P., 1983. Optimal choice of fixed and switched shunt capacitors on radial distributors by the method of local variations. IEEE transactions on power apparatus and systems, (6), pp.1607-1615.

[54] Baran, M. and Wu, F.F., 1989. Optimal sizing of capacitors placed on a radial distribution system. IEEE Transactions on power Delivery, 4(1), pp.735-743.

[55] Baran, M.E. and Wu, F.F., 1989. Optimal capacitor placement on radial distribution systems. IEEE Transactions on power Delivery, 4(1), pp.725-734.

[56] Sharaf, A.M. and Ibrahim, S.T., 1996. Optimal capacitor placement in distribution networks. Electric power systems research, 37(3), pp.181-187.

[57] Jasmon, G.B. and Lee, L.H.C.C., 1991. Distribution network reduction for voltage stability analysis and loadflow calculations. International Journal of Electrical Power \& Energy Systems, 13(1), pp.9-13.

[58] Khodr, H.M., Olsina, F.G., De Oliveira-De Jesus, P.M. and Yusta, J.M., 2008. Maximum savings approach for location and sizing of capacitors in distribution systems. Electric Power Systems Research, 78(7), pp.1192-1203.

[59] Soto, S. and Hinojosa, V., 2016. Stochastic optimal allocation of reactive power banks for system loss minimization. IEEE Latin America Transactions, 14(4), pp.1980-1987.

[60] Jannat, M.B. and Savić, A.S., 2016. Optimal capacitor placement in distribution networks regarding uncertainty in active power load and distributed generation units production. IET Generation, Transmission \& Distribution, 10(12), pp.3060-3067.

[61] Minsky, M.L., 1958, November. Some methods of artificial intelligence and heuristic programming. In Proc. Symposium on the Mechanization of Thought Processes, Teddington.

[62] Ng, H.N., Salama, M.M.A. and Chikhani, A.Y., 2000. Classification of capacitor allocation techniques. IEEE Transactions on power delivery, 15(1), pp.387-392.

[63] Abdel-Salam, T.S., Chikhani, A.Y. and Hackam, R., 1994. A new technique for loss reduction using compensating capacitors applied to distribution systems with varying load condition. IEEE Transactions on Power Delivery, 9(2), pp.819-827.

[64] Chis, M., Salama, M.M.A. and Jayaram, S., 1997. Capacitor placement in distribution systems using heuristic search strategies. IEE ProceedingsGeneration, Transmission and Distribution, 144(3), pp.225-230.

[65] da Silva, I.C., Carneiro, S., de Oliveira, E.J., de Souza Costa, J., Pereira, J.L.R. and Garcia, P.A.N., 2008. A heuristic constructive algorithm for capacitor placement on distribution systems. IEEE Transactions on Power Systems, 23(4), pp.1619-1626.

[66] Hamouda, A. and Sayah, S., 2013. Optimal capacitors sizing in distribution feeders using heuristic search based node stability-indices. International Journal of Electrical Power \& Energy Systems, 46, pp.56-64.

[67] Raju, M.R., Murthy, K.R. and Ravindra, K., 2012. Direct search algorithm for capacitive compensation in radial distribution systems. International Journal of Electrical Power \& Energy Systems, 42(1), pp.24-30.
[68] El-Fergany, A.A. and Abdelaziz, A.Y., 2014. Capacitor allocations in radial distribution networks using cuckoo search algorithm. IET Generation, Transmission \& Distribution, 8(2), pp.223-232.

[69] El-Fergany, A.A. and Abdelaziz, A.Y., 2014. Efficient heuristic-based approach for multi-objective capacitor allocation in radial distribution networks. IET Generation, Transmission \& Distribution, 8(1), pp.7080.

[70] Gnanasekaran, N., Chandramohan, S., Kumar, P.S. and Imran, A.M., 2016. Optimal placement of capacitors in radial distribution system using shark smell optimization algorithm. Ain Shams Engineering Journal, 7(2), pp.907-916.

[71] Dixit, M., Kundu, P. and Jariwala, H.R., 2018. Optimal integration of shunt capacitor banks in distribution networks for assessment of techno-economic asset. Computers \& Electrical Engineering, 71, pp.331345.

[72] Chiou, J.P. and Chang, C.F., 2015. Development of a novel algorithm for optimal capacitor placement in distribution systems. International Journal of Electrical Power \& Energy Systems, 73, pp.684-690.

[73] Devabalaji, K.R., Ravi, K. and Kothari, D.P., 2015. Optimal location and sizing of capacitor placement in radial distribution system using bacterial foraging optimization algorithm. International Journal of Electrical Power \& Energy Systems, 71, pp.383-390.

[74] Askarzadeh, A., 2016. Capacitor placement in distribution systems for power loss reduction and voltage improvement: a new methodology. IET Generation, Transmission \& Distribution, 10(14), pp.3631-3638.

[75] Muthukumar, K. and Jayalalitha, S., 2018. Multiobjective hybrid evolutionary approach for optimal planning of shunt capacitors in radial distribution systems with load models. Ain Shams Engineering Journal, 9(4), pp.1975-1988.

[76] Mujezinović, A., Turković, N., Dautbašić, N., Dedović, M.M. and Turković, I., 2019, March. Use of Integer Genetic Algorithm for Optimal Allocation and Sizing of the Shunt Capacitor Banks in the Radial Distribution Networks. In 2019 18th International Symposium INFOTEH-JAHORINA (INFOTEH) (pp. 1-6). IEEE.

[77] Kokash, N., 2005. An introduction to heuristic algorithms. Department of Informatics and Telecommunications. University of Trento.

[78] Aman, M.M., Jasmon, G.B., Bakar, A.H.A., Mokhlis, H. and Karimi, M., 2014. Optimum shunt capacitor placement in distribution system-A review and comparative study. Renewable and Sustainable Energy Reviews, 30, pp.429-439.

[79] Sundhararajan, S. and Pahwa, A., 1994. Optimal selection of capacitors for radial distribution systems using a genetic algorithm. IEEE transactions on Power Systems, 9(3), pp.1499-1507.

[80] Das, D., 2002. Reactive power compensation for radial distribution networks using genetic algorithm. International journal of electrical power \& energy systems, 24(7), pp.573-581.

[81] Haghifam, M.R. and Malik, O.P., 2007. Genetic algorithm-based approach for fixed and switchable 
capacitors placement in distribution systems with uncertainty and time varying loads. IET generation, transmission \& distribution, 1(2), pp.244-252.

[82] Levitin, G., Kalyuzhny, A., Shenkman, A. and Chertkov, M., 2000. Optimal capacitor allocation in distribution systems using a genetic algorithm and a fast energy loss computation technique. IEEE Transactions on Power Delivery, 15(2), pp.623-628.

[83] Boone, G. and Chiang, H.D., 1993. Optimal capacitor placement in distribution systems by genetic algorithm. International Journal of Electrical Power \& Energy Systems, 15(3), pp.155-161.

[84] Pazouki, S., Mohsenzadeh, A., Haghifam, M.R. and Ardalan, S., 2015. Simultaneous allocation of charging stations and capacitors in distribution networks improving voltage and power loss. Canadian Journal of Electrical and Computer Engineering, 38(2), pp.100105.

[85] Ng, H.N., Salama, M.M.A. and Chikhani, A.Y., 2000. Capacitor allocation by approximate reasoning: fuzzy capacitor placement. IEEE transactions on power delivery, 15(1), pp.393-398.

[86] Bhattacharya, S.K. and Goswami, S.K., 2009. A new fuzzy based solution of the capacitor placement problem in radial distribution system. Expert systems with applications, 36(3), pp.4207-4212.

[87] Das, D., 2008. Optimal placement of capacitors in radial distribution system using a Fuzzy-GA method. International Journal of Electrical Power \& Energy Systems, 30(6-7), pp.361-367.

[88] Gampa, S.R. and Das, D., 2016. Optimum placement of shunt capacitors in a radial distribution system for substation power factor improvement using fuzzy GA method. International Journal of Electrical Power \& Energy Systems, 77, pp.314-326.

[89] Singh, S.P. and Rao, A.R., 2012. Optimal allocation of capacitors in distribution systems using particle swarm optimization. International Journal of Electrical Power \& Energy Systems, 43(1), pp.1267-1275.

[90] Kanwar, N., Gupta, N., Swarnkar, A., Niazi, K.R. and Bansal, R.C., 2015. New sensitivity based approach for optimal allocation of shunt capacitors in distribution networks using PSO. Energy Procedia, 75, pp.11531158 .

[91] Huang, S.J., 2000. An immune-based optimization method to capacitor placement in a radial distribution system. IEEE Transactions on Power Delivery, 15(2), pp.744-749.

[92] Huang, S.J. and Liu, X.Z., 2012. A plant growth-based optimization approach applied to capacitor placement in power systems. IEEE Transactions on Power Systems, 27(4), pp.2138-2145.

[93] Pires, D.F., Martins, A.G. and Antunes, C.H., 2005. A multiobjective model for VAR planning in radial distribution networks based on tabu search. IEEE Transactions On Power Systems, 20(2), pp.1089-1094.

[94] Mendes, A., Franca, P.M., Lyra, C., Pissarra, C. and Cavellucci, C., 2005. Capacitor placement in large-sized radial distribution networks. IEE ProceedingsGeneration, Transmission and Distribution, 152(4), pp.496-502.
[95] Sultana, S. and Roy, P.K., 2014. Optimal capacitor placement in radial distribution systems using teaching learning based optimization. International Journal of Electrical Power \& Energy Systems, 54, pp.387-398.

[96] Kaur, D. and Sharma, J., 2013. Multiperiod shunt capacitor allocation in radial distribution systems. International Journal of Electrical Power \& Energy Systems, 52, pp.247-253.

[97] Carlisle, J.C. and El-Keib, A.A., 2000. A graph search algorithm for optimal placement of fixed and switched capacitors on radial distribution systems. IEEE Transactions on Power Delivery, 15(1), pp.423-428.

[98] Shuaib, Y.M., Kalavathi, M.S. and Rajan, C.C.A., 2015. Optimal capacitor placement in radial distribution system using gravitational search algorithm. International Journal of Electrical Power \& Energy Systems, 64, pp.384-397.

[99] Abu-Mouti, F.S. and El-Hawary, M.E., 2011. Optimal distributed generation allocation and sizing in distribution systems via artificial bee colony algorithm. IEEE transactions on power delivery, 26(4), pp.2090-2101.

[100] Gallego, R.A., Monticelli, A.J. and Romero, R., 2001. Optimal capacitor placement in radial distribution networks. IEEE Transactions on Power Systems, 16(4), pp.630-637.

[101] Kannan, S.M., Renuga, P., Kalyani, S. and Muthukumaran, E., 2011. Optimal capacitor placement and sizing using Fuzzy-DE and Fuzzy-MAPSO methods. Applied Soft Computing,11(8), pp.49975005.

[102] Goswami, S.K., Ghose, T. and Basu, S.K., 1999. An approximate method for capacitor placement in distribution system using heuristics and greedy search technique. Electric Power Systems Research,51(3), pp.143-151.

[103] Abril, I.P., 2017. Algorithm of inclusion and interchange of variables for capacitors placement. Electric Power Systems Research, 148, pp.117-126.

[104] Tamilselvan, V., Jayabarathi, T., Raghunathan, T. and Yang, X.S., 2018. Optimal capacitor placement in radial distribution systems using flower pollination algorithm. Alexandria engineering journal,57(4), pp.2775-2786.

[105] Abul'Wafa, A.R., 2014. Optimal capacitor placement for enhancing voltage stability in distribution systems using analytical algorithm and Fuzzy-Real Coded GA. International Journal of Electrical Power \& Energy Systems, 55, pp.246-252.

[106] Injeti, S.K., Thunuguntla, V.K. and Shareef, M., 2015. Optimal allocation of capacitor banks in radial distribution systems for minimization of real power loss and maximization of network savings using bio-inspired optimization algorithms. International Journal of Electrical Power \& Energy Systems, 69, pp.441-455.

[107] Murty, V.V.S.N. and Kumar, A., 2015. Capacitor allocation in radial distribution system with time varying ZIP load model and energy savings. Procedia Computer Science, 70, pp.377-383.

[108] da Rosa, W.M., Rossoni, P., Teixeira, J.C., Belati, E.A. and Asano, P.T.L., 2016. Optimal allocation of 
capacitor banks using genetic algorithm and sensitivity analysis. IEEE Latin America Transactions, 14(8), pp.3702-3707.

[109] Askarzadeh, A., 2016. Capacitor placement in distribution systems for power loss reduction and voltage improvement: a new methodology. IET Generation, Transmission \& Distribution, 10(14), pp.3631-3638.

[110] Prakash, D.B. and Lakshminarayana, C., 2017. Optimal siting of capacitors in radial distribution network using whale optimization algorithm. Alexandria Engineering Journal, 56(4), pp.499-509.

[111] Milosevic, B. and Begovic, M., 2004. Capacitor placement for conservative voltage reduction on distribution feeders. IEEE transactions on power delivery, 19(3), pp.1360-1367.

[112] Javadi, M.S., Nezhad, A.E., Siano, P., Shafie-khah, M. and Catalão, J.P., 2017. Shunt capacitor placement in radial distribution networks considering switching transients decision making approach. International Journal of Electrical Power \& Energy Systems, 92, pp.167-180.

[113] Baghzouz, Y. and Ertem, S., 1990. Shunt capacitor sizing for radial distribution feeders with distorted substation voltages. IEEE Transactions on Power Delivery, 5(2), pp.650-657.

[114] Wu, Z.Q. and Lo, K.L., 1995. Optimal choice of fixed and switched capacitors in radial distributors with distorted substation voltage. IEE ProceedingsGeneration, Transmission and Distribution, 142(1), pp.24-28.

[115] Yu, X.M., Xiong, X.Y. and Wu, Y.W., 2004. A PSObased approach to optimal capacitor placement with harmonic distortion consideration. Electric Power Systems Research, 71(1), pp.27-33.

[116] Masoum, M.A., Ladjevardi, M., Jafarian, A. and Fuchs, E.F., 2004. Optimal placement, replacement and sizing of capacitor banks in distorted distribution networks by genetic algorithms. IEEE transactions on power delivery, 19(4), pp.1794-1801.

[117] Masoum, M.A.S., Ladjevardi, M., Fuchs, E.F. and Grady, E.M., 2002, July. Optimal placement and sizing of fixed and switched capacitor banks under nonsinusoidal operating conditions. In IEEE Power Engineering Society Summer Meeting, (Vol. 2, pp. 807813). IEEE.

[118] Masoum, M.A.S., Ladjevardi, M., Fuchs, E.F. and Grady, W.M., 2004. Application of local variations and maximum sensitivities selection for optimal placement of shunt capacitor banks under nonsinusoidal operating conditions. International Journal of Electrical Power \& Energy Systems, 26(10), pp.761-769.

[119] Masoum, M.A., Jafarian, A., Ladjevardi, M., Fuchs, E.F. and Grady, W.M., 2004. Fuzzy approach for optimal placement and sizing of capacitor banks in the presence of harmonics. IEEE Transactions on Power Delivery, 19(2), pp.822-829.

[120] Ayoubi, M., Hooshmand, R.A. and Esfahani, M.T., 2017. Optimal capacitor placement in distorted distribution systems considering resonance constraint using multi-swarm particle swarm optimisation algorithm. IET Generation, Transmission \& Distribution, 11(13), pp.3210-3221.

[121] Othman, A.M., 2016. Optimal capacitor placement by Enhanced Bacterial Foraging Optimization (EBFO) with accurate thermal re-rating of critical cables. Electric Power Systems Research, 140, pp.671680.

[122] Vuletić, J. and Todorovski, M., 2016. Optimal capacitor placement in distorted distribution networks with different load models using Penalty Free Genetic Algorithm. International Journal of Electrical Power \& Energy Systems, 78, pp.174-182.

[123] Sajjadi, S.M., Haghifam, M.R. and Salehi, J., 2013. Simultaneous placement of distributed generation and capacitors in distribution networks considering voltage stability index. International Journal of Electrical Power \& Energy Systems, 46, pp.366-375.

[124] Mahaei, S.M., Sami, T., Shilebaf, A. and Jafarzadeh, J., 2012, May. Simultaneous placement of distributed generations and capacitors with multi-objective function. In 2012 Proceedings of 17th Conference on Electrical Power Distribution (pp. 1-9). IEEE.

[125] Mady, I.B., 2009. Optimal sizing of capacitor banks and distributed generation in distorted distribution networks by genetic algorithms. IEEE/ICEED, pp.1-4.

[126] Moradi, M.H., Zeinalzadeh, A., Mohammadi, Y. and Abedini, M., 2014. An efficient hybrid method for solving the optimal sitting and sizing problem of DG and shunt capacitor banks simultaneously based on imperialist competitive algorithm and genetic algorithm. International Journal of Electrical Power \& Energy Systems, 54, pp.101-111.

[127] Biswas, S., Goswami, S.K. and Chatterjee, A., 2014. Optimal distributed generation placement in shunt capacitor compensated distribution systems considering voltage sag and harmonics distortions. IET Generation, Transmission \& Distribution, 8(5), pp.783-797.

[128] Zeinalzadeh, A., Mohammadi, Y. and Moradi, M.H., 2015. Optimal multi objective placement and sizing of multiple DGs and shunt capacitor banks simultaneously considering load uncertainty via MOPSO approach. International Journal of Electrical Power \& Energy Systems, 67, pp.336-349.

[129] Khodabakhshian, A. and Andishgar, M.H., 2016. Simultaneous placement and sizing of DGs and shunt capacitors in distribution systems by using IMDE algorithm. International Journal of Electrical Power \& Energy Systems, 82, pp.599-607.

[130] Dixit, M., Kundu, P. and Jariwala, H.R., 2017. Incorporation of distributed generation and shunt capacitor in radial distribution system for technoeconomic benefits. Engineering Science and Technology, an International Journal, 20(2), pp.482493.

[131] Biswas, P.P., Mallipeddi, R., Suganthan, P.N. and Amaratunga, G.A., 2017. A multiobjective approach for optimal placement and sizing of distributed generators and capacitors in distribution network. Applied Soft Computing, 60, pp.268-280.

[132] Su, C.T. and Lee, C.S., 2001. Feeder reconfiguration and capacitor setting for loss reduction of distribution 
systems. Electric power systems research, 58(2), pp.97102.

[133] Jiang, D. and Baldick, R., 1996. Optimal electric distribution system switch reconfiguration and capacitor control. IEEE transactions on Power Systems, 11(2), pp.890-897.

[134] Rezaei, P., Vakilian, M. and Hajipour, E., 2011, September. Reconfiguration and capacitor placement in radial distribution systems for loss reduction and reliability enhancement. In 2011 16th International Conference on Intelligent System Applications to Power Systems (pp. 1-6). IEEE.

[135] Rong, Z., Xiyuan, P., Jinliang, H. and Xinfu, S., 2002, October. Reconfiguration and capacitor placement for loss reduction of distribution system. In 2002 IEEE Region 10 Conference on Computers, Communications, Control and Power Engineering. TENCOM'02. Proceedings. (Vol. 3, pp. 1945-1949). IEEE.

[136] Chang, C.F., 2008. Reconfiguration and capacitor placement for loss reduction of distribution systems by ant colony search algorithm. IEEE Transactions on Power Systems, 23(4), pp.1747-1755.

[137] Peponis, G.J., Papadopoulos, M.P. and Hatziargyriou, N.D., 1995. Distribution network reconfiguration to minimize resistive line losses. IEEE Transactions on Power Delivery, 10(3), pp.1338-1342.

[138] de Oliveira, L.W., Carneiro Jr, S., De Oliveira, E.J., Pereira, J.L.R., Silva Jr, I.C. and Costa, J.S., 2010. Optimal reconfiguration and capacitor allocation in radial distribution systems for energy losses minimization. International Journal of Electrical Power \& Energy Systems, 32(8), pp.840-848.

[139] Muthukumar, K. and Jayalalitha, S., 2017. Integrated approach of network reconfiguration with distributed generation and shunt capacitors placement for power loss minimization in radial distribution networks. Applied Soft Computing, 52, pp.1262-1284.

[140] Mehmood, K.K., Kim, C.H., Khan, S.U. and Haider, Z.M., 2018. Unified Planning of Wind Generators and Switched Capacitor Banks: A Multiagent ClusteringBased Distributed Approach. IEEE Transactions on Power Systems, 33(6), pp.6978-6988.

[141] Ziari, I., Ledwich, G. and Ghosh, A., 2013. A new technique for optimal allocation and sizing of capacitors and setting of LTC. International Journal of Electrical Power \& Energy Systems, 46, pp.250-257.

[142] Grainger, J.J. and Civanlar, S., 1985. Volt/var control on distribution systems with lateral branches using shunt capacitors and voltage regulators part I: The overall problem. IEEE Transactions on Power Apparatus and Systems, (11), pp.3278-3283.

[143] Civanlar, S. and Grainger, J.J., 1985. Volt/Var control on distribution systems with lateral branches using shunt capacitors and voltage regulators Part II: The solution method. IEEE transactions on power apparatus and systems, (11), pp.3284-3290.

[144] Civanlar, S. and Grainger, J.J., 1985. Volt/Var control on distribution systems with lateral branches using shunt capacitors and voltage regulators Part III: The numerical results. IEEE transactions on power apparatus and systems, (11), pp.3291-3297.
[145] Gu, Z. and Rizy, D.T., 1996. Neural networks for combined control of capacitor banks and voltage regulators in distribution systems. IEEE transactions on power delivery, 11(4), pp.1921-1928.

[146] Carpinelli, G., Noce, C., Proto, D. and Varilone, P., 2006. Voltage regulators and capacitor placement in three-phase distribution systems with non-linear and unbalanced loads. International journal of Emerging electric power systems, 7(4).

[147] Szuvovivski, I., Fernandes, T.S.P. and Aoki, A.R., 2012. Simultaneous allocation of capacitors and voltage regulators at distribution networks using genetic algorithms and optimal power flow. International Journal of Electrical Power \& Energy Systems, 40(1), pp.62-69.

[148] Franco, J.F., Rider, M.J., Lavorato, M. and Romero, R., 2013. A mixed-integer LP model for the optimal allocation of voltage regulators and capacitors in radial distribution systems. International Journal of Electrical Power \& Energy Systems, 48, pp.123-130.

[149] Hung, D.Q., Mithulananthan, N. and Bansal, R.C., 2015. A combined practical approach for distribution system loss reduction. International Journal of Ambient Energy, 36(3), pp.123-131.

[150] Abou El-Ela, A.A., El-Sehiemy, R.A. and Abbas, A.S., 2018. Optimal placement and sizing of distributed generation and capacitor banks in distribution systems using water cycle algorithm. IEEE Systems Journal, 12(4), pp.3629-3636.

[151] Saleh, A.A., Mohamed, A.A.A., Hemeida, A.M. and Ibrahim, A.A., 2019, February. Multi-Objective Whale Optimization Algorithm for Optimal Allocation of Distributed Generation and Capacitor Bank. In 2019 International Conference on Innovative Trends in Computer Engineering (ITCE) (pp. 459-465). IEEE.

[152] Laconico, K.C.C. and Aguirre, R.A., 2019, March. Optimal Load Balancing and Capacitor Sizing and Siting of an Unbalanced Radial Distribution Network. In 2019 IEEE PES GTD Grand International Conference and Exposition Asia (GTD Asia) (pp. 939944). IEEE.

[153] Arulraj, R. and Kumarappan, N., 2019. Optimal economic-driven planning of multiple DG and capacitor in distribution network considering different compensation coefficients in feeder's failure rate evaluation. Engineering Science and Technology, an International Journal, 22(1), pp.67-77.

[154] Home-Ortiz, J.M., Vargas, R., Macedo, L.H. and Romero, R., 2019. Joint reconfiguration of feeders and allocation of capacitor banks in radial distribution systems considering voltage-dependent models. International Journal of Electrical Power \& Energy Systems, 107, pp.298-310.

[155] Das, S., Das, D. and Patra, A., 2019. Operation of distribution network with optimal placement and sizing of dispatchable DGs and shunt capacitors. Renewable and Sustainable Energy Reviews, 113, p.109219.

[156] Gözel, T. and Hocaoglu, M.H., 2009. An analytical method for the sizing and siting of distributed generators in radial systems. Electric power systems research, 79(6), pp.912-918. 